\title{
Multichannel Azimuth Processing in ScanSAR and TOPS Mode Operation
}

\author{
Nicolas Gebert, Gerhard Krieger, Senior Member, IEEE, and Alberto Moreira, Fellow, IEEE
}

\begin{abstract}
Due to a system-inherent limitation, conventional synthetic aperture radar (SAR) is incapable of imaging a wide swath with high geometric resolution. This restriction can be overcome by systems with multiple receive channels in combination with an additional digital signal processing network. So far, the application of such digital beamforming algorithms for highresolution wide-swath SAR imaging has been restricted to multichannel systems in stripmap operation. However, in stripmap mode, the overall azimuth antenna length restricts the achievable swath width, thus preventing very wide swaths as requested by future SAR missions. Consequently, new concepts for ultrawideswath imaging are needed. A promising candidate is a SAR system with multiple azimuth channels being operated in burst mode. This paper analyzes innovative ScanSAR and Terrain Observation by Progressive Scans (TOPS) system concepts with regard to multichannel azimuth processing. For this, the theoretical analyses, performance figures, and SAR signal processing, which had previously been derived for multichannel stripmap mode, are extended to systems operating in burst modes. The investigations reveal that multichannel ScanSAR systems enable the imaging of ultrawide swaths with high azimuth resolution and compact antenna lengths. These considerations are embedded in a multichannel ScanSAR system design example to demonstrate its capability to image an ultrawide swath of $400 \mathrm{~km}$ with a high geometric resolution of $5 \mathrm{~m}$. In a next step, this system is adapted to TOPS mode operation, including an innovative "staircase" multichannel processing approach optimized for TOPS.
\end{abstract}

Index Terms-High-resolution ultrawide-swath synthetic aperture radar (SAR) imaging, multichannel azimuth processing, multichannel burst-mode operation, ScanSAR, TOPS.

\section{INTRODUCTION}

$\mathbf{I}$ N CONVENTIONAL synthetic aperture radar (SAR) systems, a high geometric resolution in azimuth and a wideswath coverage impose contradicting requirements on system design: A high azimuth resolution requires a large Doppler bandwidth, which has to be sampled with a sufficiently high pulse repetition frequency (PRF). In contrast, the unambiguous swath width is directly related to the separation of subsequently transmitted pulses, meaning that a required swath width limits the PRF value. This system-inherent limitation can be over-

Manuscript received March 31, 2009; revised September 18, 2009 and December 7, 2009. Date of publication March 25, 2010; date of current version June 23, 2010

N. Gebert and G. Krieger are with the Microwaves and Radar Institute, German Aerospace Center (DLR), 82234 Weßling, Germany (e-mail: nico. gebert@dlr.de).

A. Moreira is with the Microwaves and Radar Institute, German Aerospace Center (DLR), 82234 Weßling, Germany, and also with the University of Karlsruhe, 76131 Karlsruhe, Germany.

Color versions of one or more of the figures in this paper are available online at http://ieeexplore.iee.org.

Digital Object Identifier 10.1109/TGRS.2010.2041356 come by innovative SAR systems with multiple receive apertures. The basic idea is then to collect for each transmitted pulse additional samples. If the receivers are arranged in along-track direction, this directly translates into an increased effective sampling rate on receive. This allows for a higher Doppler bandwidth and thus an improved geometric resolution, or a reduced PRF on transmit enabling a wider swath can be employed without degrading the resolution. Independently of the chosen approach, the specific PRF imposes a certain azimuth antenna length which limits the illuminated Doppler spectrum to the sampling rate. In addition, in multichannel systems, the timing requirement for uniform sampling further constrains the antenna length as it has to ensure spatial sample positions that deviate not too strongly from the uniform distribution [1], [2]. As an example, the studies in [2] and [3] presented a multichannel SAR system design which enabled to image a swath of $100 \mathrm{~km}$ with $1 \mathrm{~m}$ geometric resolution in stripmap mode. This allowed for a maximum PRF of $\sim 1.4 \mathrm{kHz}$ leading to an antenna length of $11.2 \mathrm{~m}$.

Future SAR missions will require a complete and frequent coverage of the Earth with a reasonably high geometric resolution. To enable such a "mapping machine," a resolution of well below $10 \mathrm{~m}$ is required in combination with a swath of several hundred kilometers. As an example, a complete imaging of the Earth with a weekly revisit time requires a swath width of $400 \mathrm{~km}$. A timing analysis of such a system in stripmap mode operation reveals a maximum PRF around $\sim 400 \mathrm{~Hz}$, which is directly driven by the required swath width. In such a case, a suitable antenna length for stripmap operation would be on the order of $\sim 35-40 \mathrm{~m}$, which is, of course, far too long to be realized. Consequently, conventionally operated multichannel SAR systems provide a high resolution, but the antenna length limits the achievable swath width (cf. Fig. 1, left). On the other hand, burst modes, e.g., ScanSAR [4], [5], TOPS [6], or the multibeam burst mode (MBBM) [7], achieve a very wide swath by continuously switching the antenna footprint between several subswaths. In ScanSAR mode, the footprint is steered in range dimension to cover all subswaths subsequently (cf. Fig. 1, middle left). A more sophisticated burst mode is TOPS (cf. Fig. 1, middle right), where, in addition to the switching in range, a steering of the footprint in azimuth dimension is done. Both modes provide an overall swath width that consists of all subswaths. Unfortunately, this is at the cost of reduced illumination time per subswath entailing a coarsened azimuth resolution, thus excluding the capability of high-resolution imaging.

In conclusion, advanced concepts are needed for the imaging of an ultrawide swath of several hundred kilometers with 

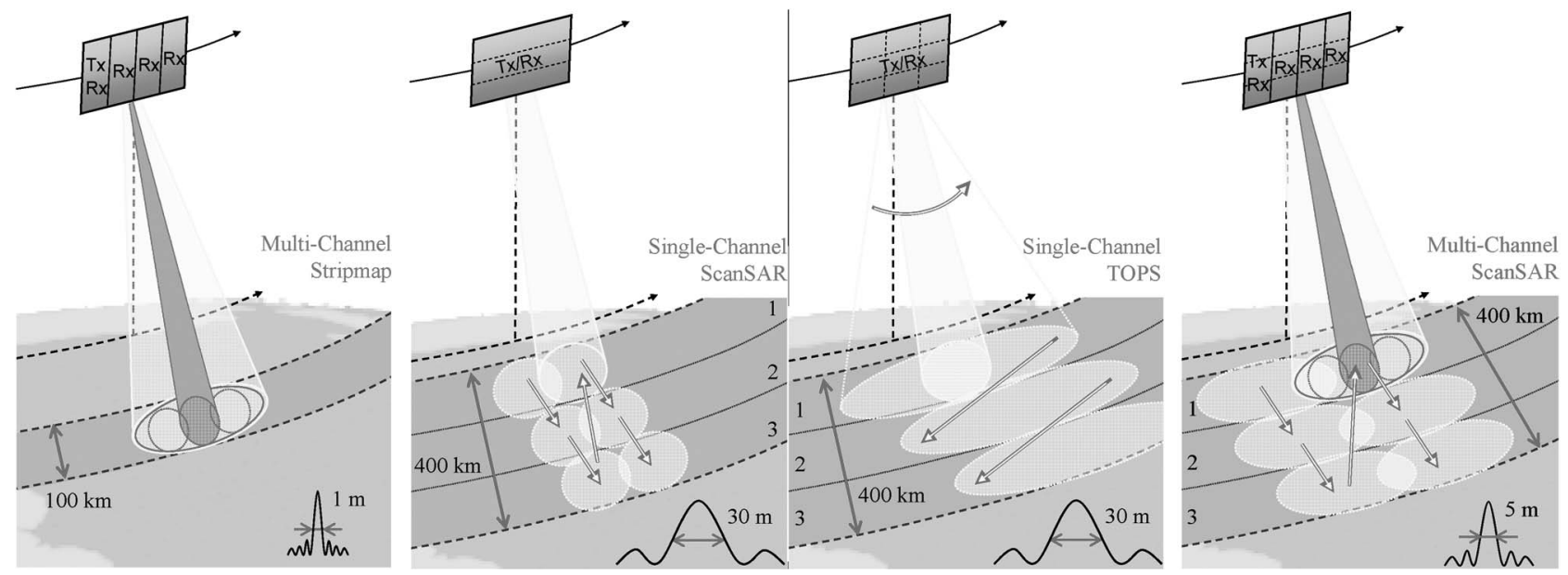

Fig. 1. (Left) Multichannel reception for high-resolution wide-swath imaging. Each Rx subaperture and the single transmitter illuminate the region marked by the bright (yellow) footprint. After digital combination of all received signals, the dark (red) pencil beam can be formed. Middle shows the ultrawide swaths obtained by burst-mode systems as ScanSAR (middle, left) and TOPS (middle, right) with effectively a single Rx and Tx aperture. The shaded (yellow) area indicates the jointly steered Tx/Rx beam, while the dotted area represents the steering range. (Right) Multichannel system in burst-mode operation (exemplarily ScanSAR) combining high resolution with ultrawide swaths.

high azimuth resolution well below $10 \mathrm{~m}$. In this regard, the innovative operation of multichannel SAR systems in burst modes was suggested in [7]-[10] and is shown in Fig. 1 on the right. A detailed investigation of the multichannel burst modes with focus on ScanSAR and a later extension to TOPS mode will be given in this paper. In the following, multichannel configurations with a single transmit ("Tx") antenna and several receiving ("Rx") antennas are considered, where Tx and Rx can be realized on separate platforms as well as separately on the same platform or even integrated in the same antenna by transmit-and-receive (T/R) module technology.

This paper is organized as follows. Section II briefly summarizes the multichannel azimuth processing, integrates the 1-D processing approach into a regular 2-D SAR scheme, and recalls the analytic expressions for the most important performance parameters in stripmap operation. Then, Section III turns the focus to burst modes and starts with recalling the timing as well as the characteristic relation between the target's position and its reflected Doppler signal spectrum. As a novelty, thorough theoretical analysis extends the analytic expressions for multichannel performance parameters to burst-mode operation. In this regard, in particular, the dependence of the reflected Doppler spectrum on the target's position will be investigated with respect to the Doppler-frequency-dependent multichannel processing network (cf. Sections III-B-E). This is followed by a system design example of an innovative multichannel ScanSAR system in Section IV which enables the imaging of a $400-\mathrm{km}$-wide swath with a geometric resolution of $5 \mathrm{~m}$. In this context, the intricate connection between multichannel system design and the various parameters becomes clear. In a next step, the multichannel TOPS mode is investigated, starting with the presentation of an adapted multichannel processing strategy which is followed by a performance analysis (Section V). This paper closes with a discussion containing an outlook on future issues like the application to reflector-based systems with a feed array and the extension to fully active SAR systems with multiple transmit apertures.

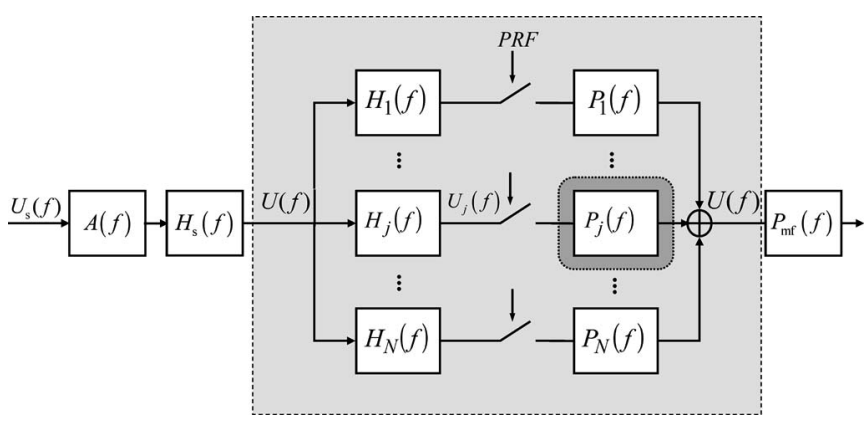

Fig. 2. Block diagram of a multichannel SAR system with "reconstruction" of $N$ subsampled channels by filters $P_{j}(f)$.

\section{Multichannel Stripmap Operation}

This section briefly recalls the multichannel reconstruction algorithm and embeds the 1-D azimuth processing into a regular 2-D processing scheme as given in Section II-B. The following sections then summarize the theoretical description of multichannel SAR performance in stripmap operation. In this context, a figure of merit for the mapping capability can be defined by the image size and its geometric resolution. The image quality is then quantified by the azimuth ambiguityto-signal ratio (AASR) and the noise-equivalent sigma zero (NESZ) which directly depends on the signal-to-noise ratio (SNR). The AASR gives a measure of how strong ambiguous contributions of a specific target disturb the image, while the NESZ describes how system noise limits the system's capability to image weakly reflecting targets.

\section{A. Multichannel Azimuth Processing}

It was shown in [1] that a multichannel SAR in azimuth can be interpreted as a linear system of filter functions which characterize the individual apertures' impulse responses in amplitude and phase in dependence on the Doppler frequency $f$. Assuming the same azimuth signal envelope pattern $A(f)$ for all $N$ channels $j$ and applying quadratic phase approximation of the azimuth signals then lead to the system model in Fig. 2. 
$U_{\mathrm{s}}(f)$ characterizes the scene, while $H_{\mathrm{s}}(f)$ is the azimuth impulse response of a single-aperture system, yielding $U(f)$ which gives the equivalent monostatic SAR signal. The functions $H_{j}(f)$ represent the channel between the transmitter (Tx) and each receiver $j\left(\mathrm{Rx}_{j}\right)$ with respect to the monostatic impulse response, resulting in the respective multichannel SAR signal $U_{j}(f)$. Assuming a single transmitter and several receiver channels, the physical along-track distance between $\mathrm{Rx}_{j}$ and $\mathrm{Tx}$ is given by $\Delta x_{j}$, while $\lambda$ represents the carrier wavelength, $R_{0}$ represents the slant range, and $v_{\mathrm{s}}$ and $v_{\mathrm{g}}$ represent the velocities of the sensor and the beam on ground, respectively

$$
H_{j}(f)=\exp \left[-\mathrm{j} \cdot \frac{v_{\mathrm{g}}}{v_{\mathrm{s}}} \cdot \frac{\pi \cdot \Delta x_{j}^{2}}{2 \cdot \lambda \cdot R_{0}}\right] \cdot \exp \left[-\mathrm{j} \cdot 2 \pi \cdot f \cdot \frac{\Delta x_{j}}{2 \cdot v_{\mathrm{s}}}\right] .
$$

After reception, each signal is sampled in azimuth by the PRF, and hence, the maximum signal bandwidth is $N \cdot P R F$ according to the effective sampling rate. A compact characterization of the whole system is then given by the matrix $\mathbf{H}(f)$, where one should note the dependence on the parameter $P R F$. See (2) at the bottom of the page.

According to a generalized sampling theorem, $N$ independent representations of a signal, each subsampled at $1 / N$ of the signal's Nyquist frequency, allow for the unambiguous "reconstruction" of the original signal from the aliased Doppler spectra of the $N$ representations. This means that any bandlimited signal $U(f)$ is uniquely determined in terms of the responses $U_{j}(f)$ or, equivalently, by the respective functions $H_{j}(f)$. This is valid independently of the spatial sample distribution as long as the samples do not coincide in space [11], [12]. Then, the inversion of $\mathbf{H}(f)$ yields a matrix $\mathbf{P}(f)$ that contains in its rows $N$ functions $P_{j}(f)$ each representing the filter for the multichannel processing of channel $j$

$$
\mathbf{P}(f)=\mathbf{H}^{-1}(f) .
$$

The original signal $U(f)$ is then recovered by filtering each channel $j$ with its appropriate "reconstruction" filter $P_{j}(f)$ and subsequent coherent combination of all weighted receiver channels (cf. Fig. 2). To complete Fig. 2, the conventional monostatic SAR focusing filter $P_{\mathrm{mf}}(f)$ is included.

\section{B. Two-Dimensional Multichannel Reconstruction}

Section II-A investigated the unambiguous reconstruction of the 1-D azimuth signal, which has to be embedded in a 2-D data reconstruction approach to derive a valid 2-D SAR processing scheme [13]. In this context, one has to consider that reconstruction of the aliased receive signals has to be applied before range cell migration correction (RCMC) as this requires a nonambiguous Doppler spectrum. This will unavoidably result in a reconstruction error, which is governed by the following two aspects: First, possible differences between the channels' range histories have to be taken into account, and second, the impact of range migration on the reconstruction is analyzed. As derived in detail in the Appendix, the different range histories and the errors introduced by applying the azimuth reconstruction subsequently for each range line are negligible in single-platform systems. Thus, a direct application of the 1-D multichannel reconstruction algorithm in a 2-D processing approach is possible to such systems without further correction steps. In multiplatform constellations, a large receiver separation might lead to a nonnegligible slant-range offset. This requires compensation which can be achieved by an appropriate range shift of the data which can, e.g., be incorporated in the range compression. After correction, multichannel reconstruction can be applied with minor errors similar to single-platform systems. In summary, the isolated 1-D consideration of the multichannel reconstruction represents a valid approximation for the application of the algorithm to 2-D data, according to the following processing flow: After range compression and-if necessary-applying a shift to each channel's data, line-byline reconstruction is carried out. After complete reconstruction of all range bins, conventional SAR processing follows, i.e., single-channel RCMC is performed, and finally, monostatic azimuth focusing results in the SAR image [13].

\section{Signal Power}

The signal power $p_{\mathrm{s}}$ is defined as the mean energy of the unambiguous azimuth signal $U(f)$, which is limited by the system bandwidth in azimuth $I_{\mathrm{S}}=N \cdot P R F$. This spectral limitation is expressed by the rectangular window function $\operatorname{rect}\left(f / I_{\mathrm{S}}\right)$, and the calculation of the mean value is indicated by the operator $\mathbf{E}[\cdot]^{1}$

$$
p_{\mathrm{S}}=\mathrm{E}\left[\left|U(f) \cdot \operatorname{rect}\left(\frac{f}{I_{\mathrm{S}}}\right)\right|^{2}\right] \text {. }
$$

Assuming azimuth focusing with a processing function of unity gain on a defined Doppler bandwidth $B_{\mathrm{D}} \leq I_{\mathrm{S}}$, the signal power is described by $p_{\mathrm{s}, B_{\mathrm{D}}}$, where the mean value is still calculated on the original interval $I_{\mathrm{S}}$

$$
p_{\mathrm{s}, B_{\mathrm{D}}}=\mathrm{E}\left[\left|U(f) \cdot \operatorname{rect}\left(\frac{f}{B_{\mathrm{D}}}\right)\right|^{2}\right], \quad B_{\mathrm{D}} \leq I_{\mathrm{S}} .
$$

\footnotetext{
${ }^{1}$ For deterministic signals, the mathematical operator $\mathbf{E}[\cdot]$ is identical to an integration over the interval $I_{\mathrm{S}}$, normalized by its width $N \cdot P R F$ or the respective number of samples. For stochastic processes, $\mathbf{E}[\cdot]$ represents the expectation value. As in both cases $\mathbf{E}[\cdot]$ gives a measure of the power the same operator symbol is used.
}

$$
\mathbf{H}(f)=\left[\begin{array}{ccc}
H_{1}(f) & \cdots & H_{N}(f) \\
H_{1}(f+P R F) & \cdots & H_{N}(f+P R F) \\
\vdots & \ddots & \vdots \\
H_{1}(f+(N-1) \cdot P R F) & \cdots & H_{N}(f+(N-1) \cdot P R F)
\end{array}\right]
$$




\section{Residual Reconstruction Error and Azimuth Ambiguities}

As discussed in [2], energy outside the band $I_{\mathrm{S}}=$ $[-N \cdot P R F / 2, N \cdot P R F / 2]$ of the original signal spectrum is not cancelled by the algorithm and disturbs the unambiguous reconstruction of the multichannel SAR signal. After focusing, this will give rise to azimuth ambiguities in the SAR image, which are governed by the aliasing components of the signal and the weighting by the reconstruction filter functions $P_{j}(f)$. The system band $I_{\mathrm{S}}$ is split into $N$ intervals $I_{m}$ on which the filters $P_{j}(f)$ are defined. In order to calculate the residual ambiguities, all subbands $I_{m}$ with $m>m_{0}$, where the signal is not properly reconstructed, have to be included. The complete ambiguous energy due to aliasing $p_{\mathrm{a}}$ is obtained by considering all individual ambiguous contributions after reconstruction, where the factor two accounts for both signs of $k$. The index $k$ indicates a shift by $f=k \cdot P R F$ caused by the sampling. Note that $P_{j}(f)$ varies with the PRF, thus introducing a dependence of the ambiguous power on this parameter

$p_{\mathrm{a}}=\mathrm{E}\left[\left|2 \cdot \sum_{k=1}^{\infty}\left(U_{k}(f) \cdot \sum_{m=m_{0}}^{N} \sum_{j=1}^{N} H_{j k}(f) \cdot P_{j m}(f)\right)\right|^{2}\right]$.

After focusing, the respective processed Doppler bandwidth $B_{\mathrm{D}}$ and the associated low-pass filtering are included, yielding the ambiguous power in the image $p_{\mathrm{a}, B_{\mathrm{D}}}$

$$
\begin{array}{r}
p_{\mathrm{a}, B_{\mathrm{D}}}=E\left[\mid 2 \cdot \sum_{k=1}^{\infty}\left(U_{k}(f) \cdot \sum_{m=m_{0}}^{N} \sum_{j=1}^{N} H_{j k}(f) \cdot P_{j m}(f)\right)\right. \\
\left.\left.\operatorname{rect}\left(\frac{f}{B_{\mathrm{D}}}\right)\right|^{2}\right] \cdot
\end{array}
$$

The AASR $\left(A A S R_{N}\right)$ is then obtained straightforward by combining (7) with (5).

\section{E. SNR Scaling Factor $\Phi_{b f}$ and NESZ}

The reconstruction algorithm is based on an inverse network of filters. Depending on the spatial sample distribution defined by sensor velocity $v_{\mathrm{s}}$, antenna dimensions, and PRF, this might result in a degradation of the SNR. This influence of the digital processing network on signal and noise power is quantified by the SNR scaling factors $\Phi_{\mathrm{bf}}$ and $\Phi_{\mathrm{bf}, B_{\mathrm{D}}}$, which refer to the data before and after focusing with a Doppler bandwidth of $B_{\mathrm{D}}$, respectively (cf. Fig. 3).

Assuming thermal noise to be the dominant source, the variation of the SNR normalized to the value obtained for uniform sampling: i.e., $P R F=P R F_{\text {uni }}$, has been derived in detail in [3] and is given in (8). Note that this expression is valid not only for the multichannel reconstruction algorithm

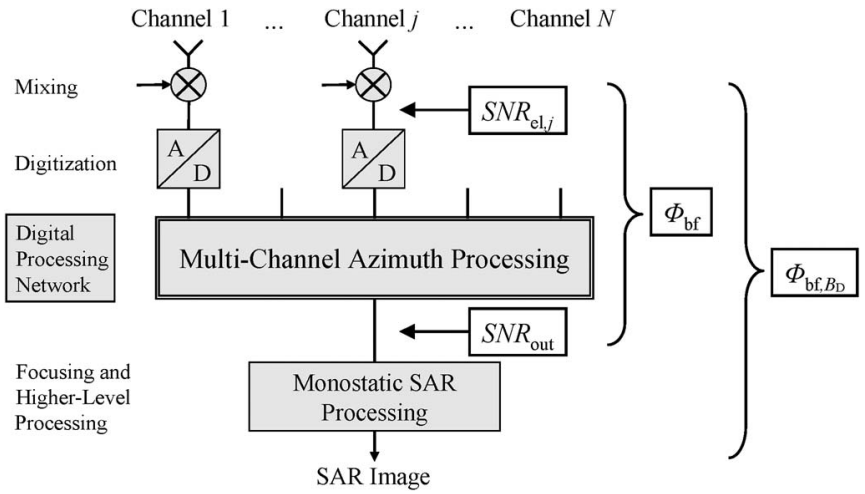

Fig. 3. Azimuth processing block diagram giving the SNR scaling factors before $\left(\Phi_{\mathrm{bf}}\right)$ and after focusing $\left(\Phi_{\mathrm{bf}, B_{\mathrm{D}}}\right)$ of the SAR image.

but also for arbitrary filter functions $P_{j}(f)$. Again, the filter's dependence on the PRF should be kept in mind.

$$
\Phi_{\mathrm{bf}}:=\frac{S N R_{\mathrm{el}} / S N R_{\mathrm{out}}}{\left.\left(S N R_{\mathrm{el}} / S N R_{\mathrm{out}}\right)\right|_{P R F_{\mathrm{uni}}}}=N \cdot \sum_{j=1}^{N} \mathrm{E}\left[\left|P_{j}(f)\right|^{2}\right] .
$$

Taking into account the focusing of the data, the SNR scaling factor of the image, i.e., $\Phi_{\mathrm{bf}, B_{\mathrm{D}}}$, is obtained. Assuming a simplified model where the signal power is completely contained within the spectral band $B_{\mathrm{D}}$, the variation of the SNR in the SAR image induced by the multichannel processing is given as follows:

$$
\Phi_{\mathrm{bf}, B_{\mathrm{D}}}=N \cdot \sum_{j=1}^{N} \mathrm{E}\left[\left|P_{j}(f) \cdot \operatorname{rect}\left(\frac{f}{B_{\mathrm{D}}}\right)\right|^{2}\right] .
$$

In a final step, the well-known expression for NESZ in SAR (cf. [14] and [15]) is adapted to a multichannel system by adding the SNR scaling factor of the reconstruction filter network $\Phi_{\mathrm{bf}, B_{\mathrm{D}}}$. Note that $\Phi_{\mathrm{bf}, B_{\mathrm{D}}}$ only describes the relation between the output and input SNRs at the respective PRF comprising a possible gain by oversampling in the Doppler domain. In contrast, it does not consider a possible variation of $S N R_{\mathrm{el}}$ with the PRF. To account for this, an additional factor $\Phi_{\mathrm{NESZ}}$ might become necessary. Assuming, e.g., a constant duty cycle, the signal power remains constant as-considering exemplarily a rising PRF- the decreased energy per sample is compensated by the increased number of samples per time. In addition, the noise energy per sample remains constant, meaning that the noise power rises according to the PRF. As $\Phi_{\mathrm{bf}, B_{\mathrm{D}}}$ is normalized to the uniform case, one obtains $\Phi_{\mathrm{NESZ}}=P R F / P R F_{\mathrm{uni}}$, which directly represents the degradation of the input SNR per sample with increasing PRF. For a constant processed Doppler bandwidth, this "loss" is directly compensated by the obtained "gain" due to a higher oversampling, as mentioned earlier. This leads to the expression (see (10), shown at the bottom of the page) for the image NESZ in multichannel SAR, taking into

$$
N E S Z=\frac{256 \cdot \pi^{3} \cdot R_{0}^{3}\left(\Theta_{\mathrm{i}}\right) \cdot v_{\mathrm{s}} \cdot \sin \left(\Theta_{\mathrm{i}}\right) \cdot \mathrm{k} \cdot T \cdot B_{\mathrm{rg}}\left(\Theta_{\mathrm{i}}\right) \cdot \Phi_{\mathrm{bf}, B_{\mathrm{D}}} \cdot \Phi_{\mathrm{NESZ}} \cdot L \cdot F \cdot L_{\mathrm{az}}}{P_{\mathrm{tx}} \cdot G_{\mathrm{tx}}\left(\Theta_{\mathrm{i}}\right) \cdot N \cdot G_{\mathrm{rx}, j}\left(\Theta_{\mathrm{i}}\right) \cdot \lambda^{3} \cdot c_{0} \cdot d c}
$$


account the possible effect of a digital processing: ${ }^{2}$ with the transmitted peak power $P_{\mathrm{tx}}$, the incident angle $\Theta_{\mathrm{i}}$, the transmit antenna gain $G_{\mathrm{tx}}$, the chirp bandwidth $B_{\mathrm{rg}}$, and the duty cycle $d c$ defined by $P R F \cdot \tau$. The reflected signal is then received by $N$ apertures of gain $G_{\mathrm{rx}, j}$, each. $L$ accounts for all power losses (ohmic, system, atmospheric, etc.), while $L_{\mathrm{az}}$ gives the azimuth loss factor [17]. The thermal noise power is quantified by the receiver noise figure $F$ and $\mathrm{k} \cdot T \cdot B_{\mathrm{rg}}$, where $\mathrm{k}$ is Boltzmann's constant and $T$ is the system's equivalent noise temperature. Note that the effective sampling to determine the overall number of focused pulses is given by $N \cdot P R F$, while the duty cycle $d c$ contains only $P R F \cdot \tau$. In consequence, an increased signal power by a factor of $N$ with respect to a singlechannel system is obtained.

\section{Multichannel Burst-Mode Operation}

\section{A. ScanSAR Timing}

In order to achieve a required azimuth resolution $\delta_{\mathrm{az}}$, the necessary illumination time $T_{\mathrm{B}, i}$ or corresponding Doppler bandwidth $B_{\mathrm{B}}$ of a single burst is given in the following equation. The index " $i$ " accounts for the varying beam velocity and mean slant range of the respective subswath and the consequentially varying illumination time for a constant $B_{\mathrm{B}}$.

$$
T_{\mathrm{B}, i}=\frac{\lambda \cdot R_{0, i} \cdot B_{\mathrm{B}}}{2 \cdot v_{\mathrm{s}} \cdot v_{\mathrm{g}, i}} \approx \frac{\lambda \cdot R_{0, i} \cdot 0.89}{2 \cdot v_{\mathrm{s}} \cdot \delta_{\mathrm{az}}} .
$$

As the dependence on the subswath is governed by $R_{0, i}$ while $v_{\mathrm{g}, i}$ varies nearly negligibly, the dependence of the beam velocity will not be explicitly expressed in the following. Nevertheless, the varying $v_{\mathrm{g}}$ is accounted for in the performed simulations.

Next, the cycle time $T_{\mathrm{C}}$ that defines the time between two subsequent illuminations of the same subswath is simply determined by the sum of all burst times

$$
T_{\mathrm{C}}=\sum_{i=1}^{N_{\mathrm{B}}} T_{B, i} .
$$

In addition, the timing has to ensure for every single target a continuous illumination of time $T_{\mathrm{B}, i}$ by the azimuth antenna beam which is given by the angle $\Theta_{\mathrm{az}, i}$, as expressed by (13). In other words, $\Theta_{\mathrm{az}, i}$ represents the maximum angle under which signals from subswath $i$ are received, and thus, it defines the azimuth system bandwidth.

$$
T_{\mathrm{C}}=\frac{R_{0} \cdot \Theta_{\mathrm{az}, i}}{v_{\mathrm{g}}}-T_{\mathrm{B}, i} .
$$

Combining (12) and (13) and replacing $\Theta_{\mathrm{az}, i}$ by the corresponding bandwidth $B_{\mathrm{D}, i}$ allow for relating the overall azimuth

\footnotetext{
${ }^{2}$ This assumes a scene of distributed scatterers, imposing the velocity $v_{\mathrm{S}}$ to relate illumination time and synthetic aperture length with regard to the received signal power. The beam velocity on ground $v_{\mathrm{g}}$ would be appropriate for pointlike targets [16]. As $v_{\mathrm{g}}<v_{\mathrm{s}}$ holds true, this provides a lower bound for the signal power, and consequently, (10) provides a worst case estimation of the NESZ.
}

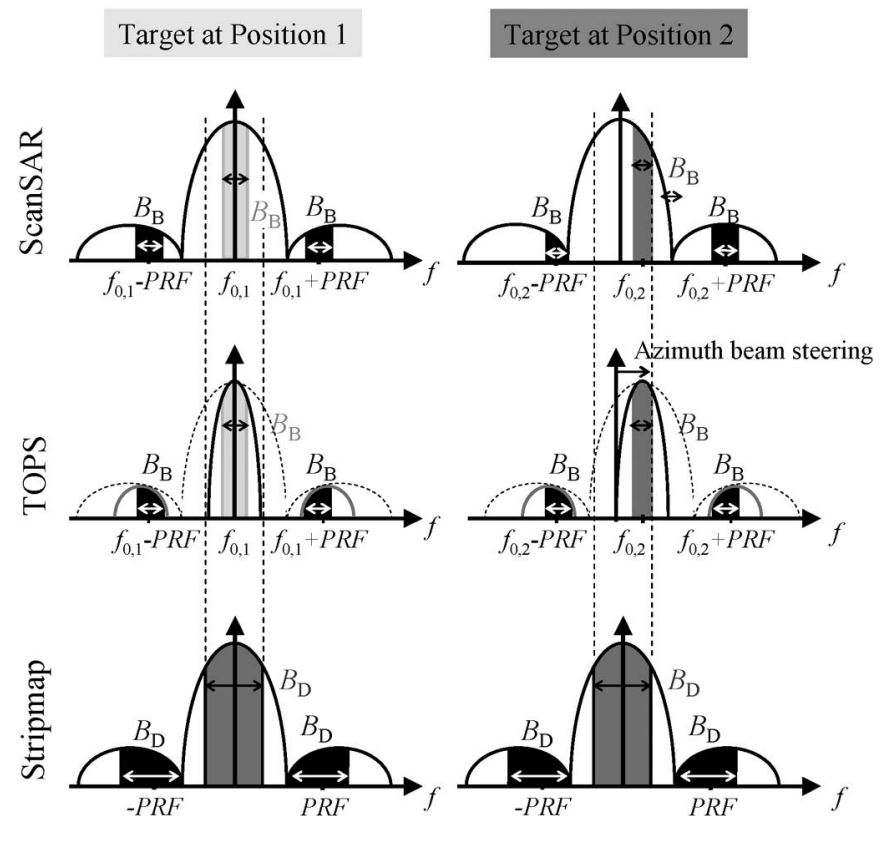

Fig. 4. Target signal spectrum (Doppler domain) in (top) ScanSAR mode and (middle) TOPS mode for two targets at different positions. For TOPS, the snapshot at a specific time is given in dashed style, while the effectively resulting envelopes for signal and ambiguities are given by solid lines and consider the weighting by the shrunk pattern. ${ }^{3}$ The respective band of a specific target depends on its position that entails a center frequency $f_{0, \mathrm{t}}$. Respective ambiguous bands are marked in black. (Bottom) For comparison, the spectrum for stripmap mode is shown.

bandwidth of subswath $i$, i.e., $B_{\mathrm{D}, i}$, with the burst bandwidth

$$
\begin{aligned}
B_{\mathrm{D}, i} & =\frac{2 \cdot v_{\mathrm{s}}}{\lambda} \cdot \sin \left(\Theta_{\mathrm{az}, i}\right) \approx \frac{2 \cdot v_{\mathrm{s}} \cdot v_{\mathrm{g}}}{\lambda \cdot R_{0}} \cdot\left(T_{\mathrm{C}}+T_{\mathrm{B}, i}\right) \\
& =B_{\mathrm{B}}+\frac{2 \cdot v_{\mathrm{s}} \cdot v_{\mathrm{g}}}{\lambda \cdot R_{0}} \cdot T_{\mathrm{C}} .
\end{aligned}
$$

If $T_{\mathrm{C}}$ is approximated by a mean burst time $T_{\mathrm{B}}$ and $N_{\mathrm{B}}$ represents the number of bursts, the following simplified expression can be derived:

$$
B_{\mathrm{D}}=\left(N_{\mathrm{B}}+1\right) \cdot B_{\mathrm{B}}
$$

Note that $B_{\mathrm{D}}$ and $B_{\mathrm{B}}$ are connected by a factor $N_{\mathrm{B}}+1$, although the system bandwidth is distributed to only $N_{\mathrm{B}}$ bursts, i.e., the overall swath compared to a stripmap system will be increased by a factor of $N_{\mathrm{B}}$ while the resulting coarsening of the azimuth resolution will be in the order of $N_{\mathrm{B}}+1$.

\section{B. Burst-Mode Target Signal Spectrum}

It is well known that the target signal spectrum depends on the target's position as this defines the angle under which the target is illuminated by the antenna (cf. Fig. 4). In

\footnotetext{
${ }^{3}$ In contrast to ScanSAR and stripmap modes, the Doppler signal envelope cannot be given straightforward for TOPS as it depends also on azimuth time. For the time-Doppler frequency characteristic in TOPS, see Fig. 13, which incorporates the characteristic shrunk pattern due to the azimuth beam steering of TOPS.
} 
Doppler Spectrum of Processing Filters

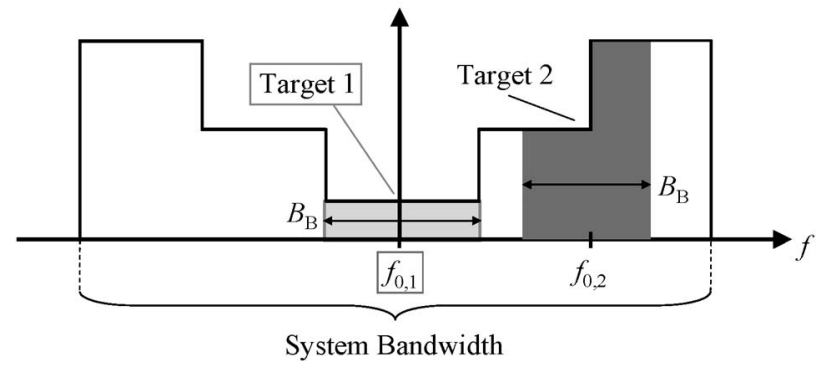

Fig. 5. Doppler spectrum (magnitude) of multichannel processing functions and spectral bands covered by targets at two different positions, namely, (light gray) " 1 ” and (dark gray) " 2 ," respectively.

consequence, targets at different positions yield echoes with different Doppler characteristics, both for ScanSAR and TOPS. This means that the target position within the burst can be "translated" to a "target center frequency" denoted by $f_{0, \mathrm{t}}$ that represents the mean frequency of the Doppler spectrum of width $B_{\mathrm{B}}$ reflected by the target during a burst. To provide a consistent notation in the following, $f_{0, t}$ will be used when referring to the position-dependent characteristics in burst-mode operation.

The maximum $f_{0, t}$ is obtained for targets at the outermost position within the antenna main beam. Taking into account the signal bandwidth $B_{\mathrm{B}}$ and the equivalent azimuth bandwidth $B_{\mathrm{D}, i}$ of subswath $i$, the respective maximum $f_{0, \mathrm{t}}$ is expressed by (16). Note that the index $i$ is omitted for reasons of succinct notation while keeping in mind the dependence on the respective subswath.

$$
\pm f_{0, \mathrm{t}, \text { max }}= \pm \frac{B_{\mathrm{D}, i}}{2} \mp \frac{B_{\mathrm{B}}}{2} .
$$

In a further step, the multichannel processing of the burstmode data is investigated. The magnitude of the Doppler spectrum of the reconstruction filter functions $P_{j}(f)$-exemplarily for a nonoptimum PRF-is shown in Fig. 5. The spectrum shows a staircase-like behavior and is subdivided into $N$ subbands of different amplitudes, which might vary strongly. In general, the more the spatial sampling in azimuth becomes nonuniform, the more the variations become strong. Considering the target signal spectra derived earlier, it becomes obvious that - in contrast to stripmap operation-ambiguities and noise disturbing the respective target are "weighted" differently by the respective multichannel reconstruction while ensuring an unchanged desired signal. In consequence, the performance figures become dependent on the target position.

\section{Burst-Mode Signal Power}

The signal power in ScanSAR operation is governed by the illumination time $T_{\mathrm{B}}$, resulting in a bandwidth $B_{\mathrm{B}}$. In addition, signal power varies with the target position represented by the target center frequency $f_{0, \mathrm{t}}$ ("scalloping") according to the respective illumination intensity, as shown in Fig. 4, top. Consequently, the received spectrum $B_{\mathrm{S}}$ of a pointlike target is characterized by a bandwidth $B_{\mathrm{B}}$ around a varying center frequency $f_{0, t}{ }^{4}$

$$
B_{\mathrm{s}}=\left[f_{0, \mathrm{t}}-\frac{B_{\mathrm{B}}}{2}, f_{0, \mathrm{t}}+\frac{B_{\mathrm{B}}}{2}\right] .
$$

The corresponding signal power $p_{\mathrm{s}, B_{s}}$ resulting from the respective band $B_{\mathrm{s}}$ can then be expressed by

$$
p_{\mathrm{s}, B_{\mathrm{s}}}=\mathrm{E}\left[\left|U(f) \cdot W\left(B_{\mathrm{s}}\right)\right|^{2}\right]
$$

where $W\left(B_{\mathrm{s}}\right)$ represents a rectangular window function confining the Doppler spectrum $B_{\mathrm{s}}$. In the following, basically all relations derived for stripmap imaging can be directly transferred to ScanSAR mode by replacing the dependence on $B_{\mathrm{D}}$ by the respective band(s) $B_{\mathrm{s}, i}$. In this context, it should be kept in mind that this introduces an additional dependence on the target position, which is represented by $f_{0, t}$.

Furthermore, it should be noted that the aforementioned considerations are valid for the ScanSAR mode. Regarding the TOPS mode, the scalloping is mitigated as only the antenna characteristics of the single elements used to effectuate the azimuth steering have to be considered when evaluating the loss of peak power with varying target position [18]. In addition, the effectively shrunk pattern caused by the steering will affect the signal power.

\section{Azimuth Ambiguities in Burst-Mode Operation: $A A S R_{N, \mathrm{~B}}$}

As presented in Section II-D, the residual azimuth ambiguities in the SAR image are governed by the aliasing contributions in the originally received signal in combination with a possible amplification due to the weighting by the reconstruction filter functions $P_{j}(f)$ of the digital processing network. Consequently, (7) is modified replacing the dependence on $B_{\mathrm{D}}$ by $W\left(B_{\mathrm{s}}\right)$, which restricts the spectrum to the burst-mode signal. One obtains the following expression for the azimuth ambiguous power $p_{\mathrm{a}, B_{\mathrm{s}}}$ resulting from the band $B_{\mathrm{s}}$ :

$$
\begin{array}{r}
p_{\mathrm{a}, B_{\mathrm{s}}}=E\left[\mid 2 \cdot \sum_{k=1}^{\infty}\left(U_{k}(f) \cdot \sum_{m=1}^{N} \sum_{j=1}^{N} H_{j k}(f) \cdot P_{j m}(f)\right)\right. \\
\left.\left.\cdot W\left(B_{\mathrm{s}}\right)\right|^{2}\right] .
\end{array}
$$

The ratio of the respectively considered signal and ambiguous powers then yields the AASR in burst mode $A A S R_{N, \mathrm{~B}}$ according to

$$
A A S R_{N, \mathrm{~B}}=\frac{p_{\mathrm{a}, B_{\mathrm{s}}}}{p_{\mathrm{s}, B_{\mathrm{s}}}} .
$$

Hence, besides the well-known varying signal power (scalloping), the use of different subspectra corresponding to

\footnotetext{
${ }^{4}$ Depending on the timing, multiple bursts from the same target may be received, e.g., to obtain multiple looks. In the following, exemplarily, only a single burst will be considered, keeping in mind that, for multiple bursts, the derived expressions can be easily extended by a simple summation over the individual bursts.
} 
TABLE I

REQUIREMENTS AND CONSTRAINTS FOR A MULTICHANNEL SCANSAR SYSTEM

\begin{tabular}{lcc}
\hline \hline \multicolumn{1}{c}{ Parameter } & Symbol & Value \\
\hline Carrier wavelength & $\lambda$ & $3.1 \mathrm{~cm}$ \\
Swath width on ground & $W_{\mathrm{g}}$ & $\geq 400 \mathrm{~km}$ \\
Geometric resolution in azimuth & $\delta_{\mathrm{az}}$ & $\leq 5 \mathrm{~m}$ \\
Azimuth ambiguity-to-signal ratio (distributed targets) & $A A S R_{N, \mathrm{~B}}$ & $\leq-20 \mathrm{~dB}$ \\
Covered incident angle range & $\Theta_{\mathrm{i}}$ & $19^{\circ}-47^{\circ}$ \\
Transmit duty cycle & $d c$ & $15 \%$ \\
\hline \hline
\end{tabular}

different target positions within the burst will result in a variation of the residual azimuth ambiguities of these targets. This yields a scallopinglike effect for the $A A S R_{N, \mathrm{~B}}$ since it varies with $f_{0, \mathrm{t}}$ as can be seen by the dependence of the ambiguous power on $B_{\mathrm{s}}$ [cf. (19)]. Although (19) is not valid for TOPS, also, this mode will suffer a scaling depending on the target position due to the frequency dependence of the filters $P_{j m}(f)$.

\section{E. SNR Scaling in Burst-Mode Operation: $\Phi_{b f, B}$}

Equivalent to the $A A S R_{N, \mathrm{~B}}$, the resulting SNR is governed by the considered parts of the Doppler spectrum given by the band(s) $B_{\mathrm{s}}$ that determine the signal power $p_{\mathrm{s}, B_{\mathrm{s}}}$ according to (18) and Section III-C. The noise scaling both in ScanSAR and TOPS is expressed by (21), where the input noise power is denoted by $p_{\mathrm{n}, \mathrm{el}}$ while $p_{\mathrm{n}, B_{\mathrm{s}}}$ describes the output noise power. The Doppler band $B_{\mathrm{S}}$ is defined by the specific target position by this introducing a scalloping with the variation of the subband.

$$
\frac{p_{\mathrm{n}, B_{\mathrm{s}}}}{p_{\mathrm{n}, \mathrm{el}}}=N \cdot F \cdot \sum_{j=1}^{N} \mathrm{E}\left[\left|P_{j}(f)\right|^{2} \cdot W\left(B_{\mathrm{s}}\right)\right] .
$$

\section{System Design EXAMPLE-MultichannEL SCANSAR}

This section gives a ScanSAR system design example to demonstrate the applicability and potentials of multichannel signal processing in burst-mode operation for ultrawide-swath imaging with high geometric resolution [8]. Note that the design was chosen exemplarily and that the derived results can be transferred to any burst-mode system, e.g., TOPS (cf. Section V) or the innovative MBBM concept, which is based on multiple transmit beams enabling the simultaneous acquisition of multiple subswaths within the same burst [7].

\section{A. Timing and System Parameters}

In the following, an X-band system is designed to image a swath of $400 \mathrm{~km}$ with an azimuth resolution below $5 \mathrm{~m}$, which exceeds, by far, the imaging capabilities of current spaceborne SAR systems. All requirements and system constraints are summarized by Table I.

At first, a timing analysis is performed. This yields four subswaths of length $105 \mathrm{~km}$ each with an orbit height of $630 \mathrm{~km}$ to cover the swath of $400 \mathrm{~km}$ (cf. Fig. 6). The necessary PRF values vary only from 1150 up to $1240 \mathrm{~Hz}$, representing a quite

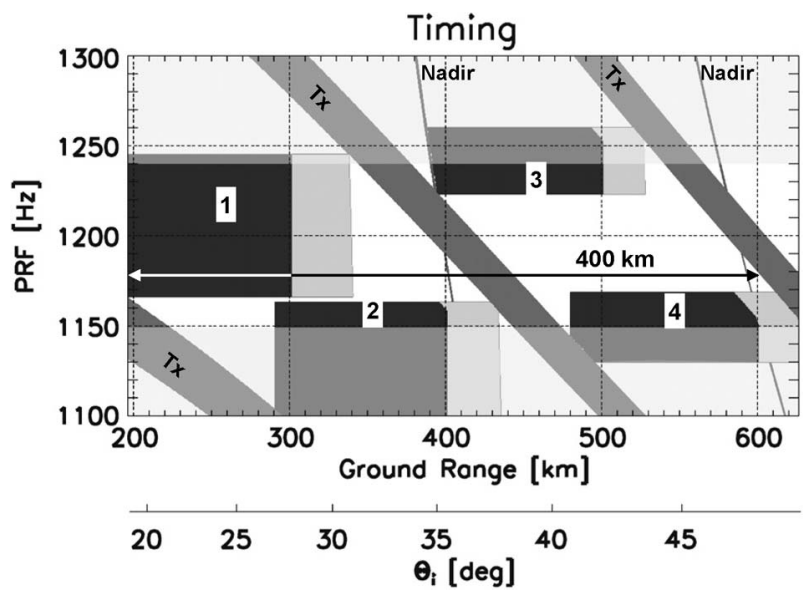

Fig. 6. Timing diagram. Depending on the applied PRF and ground range, signal reception is only possible when no transmit events ("Tx") and nadir returns ("Nadir") are interfering. In the given case, four swaths of length $105 \mathrm{~km}$ each cover a ground range of $400 \mathrm{~km}$.

good-natured case. Note that orbit height variations might result in a modified timing, but this can be compensated by a simple roll maneuver of the satellite, thus still ensuring a coverage of $400 \mathrm{~km}$ but with a different incident angle range. If a mapping of the same incident angle range is required, a possible larger PRF range is needed. In such a case, optimization strategies as presented in [2] and [3] can be applied to enable the increased PRF range.

An overall length of the antenna in azimuth of $12.8 \mathrm{~m}$ is chosen, yielding a $P R F_{\text {uni }}$ of $\sim 1180 \mathrm{~Hz}$ which is within the PRF range of operation. Furthermore, a Doppler bandwidth of $1.24 \mathrm{kHz}$ for a single burst ensures a resolution of $5 \mathrm{~m}$ for single-look processing. According to (11) and (14), this yields the respective illumination times $T_{\mathrm{B}, i}$ and Doppler bandwidths $B_{\mathrm{D}, i}$ as given in Table II. Note that the fixed burst bandwidth, in combination with the varying slant ranges of the subswaths, leads to a variation of these two parameters (cf. Table II).

In combination with the minimum PRF, a maximum overall Doppler bandwidth of $\sim 6.8 \mathrm{kHz}$ requires a minimum number of six receive apertures to fulfill the Nyquist criterion according to

$$
N \geq \frac{\max _{i}\left\{B_{\mathrm{D}, i}\right\}}{P R F_{\min }} .
$$

In the case of a ScanSAR system, it was observed that a low oversampling might introduce a tradeoff between scalloping and azimuth ambiguity suppression, as one parameter can only be improved by degrading the other according to the chosen antenna size. This conflict can be relaxed by ensuring sufficiently high oversampling, as this allows for smaller antennas in order to improve scalloping while the ambiguous energy suppression is mainly achieved by the oversampling. The drawbacks of such a solution are, of course, increased hardware costs and complexity and also a higher data rate, as more independent receiving channels are required. In the following, $N=8$ is chosen to adapt the single receive aperture size to $B_{\mathrm{D}, i}$ and to guarantee oversampling in the Doppler domain. Finally, the transmit antenna length in azimuth is set to $2.1 \mathrm{~m}$, as this is large 
TABLE II

Parameters of DifFERENT Subswaths

\begin{tabular}{lccccc}
\hline \hline \multicolumn{1}{c}{ Parameter } & Symbol & \multicolumn{4}{c}{ Value } \\
& & Subswath 1 & Subswath 2 & Subswath 3 & Subswath 4 \\
\hline Pulse repetition frequency & $P R F$ & $1180 \mathrm{~Hz}$ & $1160 \mathrm{~Hz}$ & $1240 \mathrm{~Hz}$ & $1150 \mathrm{~Hz}$ \\
Mean incident angle & $\Theta_{\mathrm{i}, \text { mean }}$ & $23.5^{\circ}$ & $31.2^{\circ}$ & $38.0^{\circ}$ & $43.9^{\circ}$ \\
Mean swath slant range & $R_{0, i}$ & $681 \mathrm{~km}$ & $725 \mathrm{~km}$ & $778 \mathrm{~km}$ & $840 \mathrm{~km}$ \\
ScanSAR burst duration & $T_{\mathrm{B}, \mathrm{i}}$ & $0.255 \mathrm{~s}$ & $0.271 \mathrm{~s}$ & $0.291 \mathrm{~s}$ & $0.314 \mathrm{~s}$ \\
Doppler bandwidth & $B_{\mathrm{D}, i}$ & $6777 \mathrm{~Hz}$ & $6440 \mathrm{~Hz}$ & $6077 \mathrm{~Hz}$ & $5713 \mathrm{~Hz}$ \\
Max. center Doppler & $f_{0, \mathrm{t}, \max }$ & $2765 \mathrm{~Hz}$ & $2597 \mathrm{~Hz}$ & $2417 \mathrm{~Hz}$ & $2236 \mathrm{~Hz}$ \\
\hline \hline
\end{tabular}

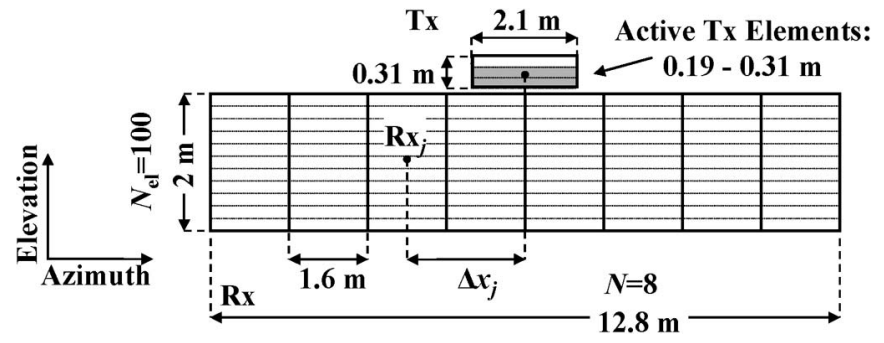

Fig. 7. Illustration of system architecture and antenna dimensions.

TABLE III

Multichannel Burst-Mode System Parameters

\begin{tabular}{lcc}
\hline \hline \multicolumn{1}{c}{ Parameter } & Symbol & Value \\
\hline Orbit height & $h_{\mathrm{s}}$ & $630 \mathrm{~km}$ \\
Sensor velocity & $v_{\mathrm{s}}$ & $7545 \mathrm{~m} / \mathrm{s}$ \\
Subswath width on ground & $W_{\mathrm{g}, \mathrm{sub}}$ & $105 \mathrm{~km}$ \\
Number of subswaths/bursts & $N_{\mathrm{B}}$ & 4 \\
Rx subapertures in azimuth & $N$ & 8 \\
Rx subaperture length in azimuth & $d_{\mathrm{az}, \mathrm{rx}}$ & $1.6 \mathrm{~m}$ \\
Tx antenna length in azimuth & $d_{\mathrm{az}, \mathrm{tx}}$ & $2.1 \mathrm{~m}$ \\
Scan cycle time & $T_{\mathrm{C}}$ & $1.1 \mathrm{~s}$ \\
Burst bandwidth & $B_{\mathrm{B}}$ & $1.24 \mathrm{kHz}$ \\
Processed azimuth bandwidth & $B_{\mathrm{D}, i}$ & $\sim 5.7 \mathrm{kHz}-6.8 \mathrm{kHz}$ \\
Tx antenna size in elevation & $d_{\mathrm{ev}, \mathrm{tx}}$ & $0.19 \mathrm{~m}-0.31 \mathrm{~m}$ \\
Overall Rx antenna height in elevation & $h_{\mathrm{ev}, \mathrm{rx}}$ & $2 \mathrm{~m}$ \\
Maximum Tx antenna gain & $G_{\mathrm{tx} . \mathrm{max}}$ & $37.2 \mathrm{~dB}-39.3 \mathrm{~dB}$ \\
Overall Rx antenna gain & $G_{\mathrm{rx}}=N \cdot G_{\mathrm{rx}, \mathrm{j}}$ & $55.3 \mathrm{~dB}$ \\
Tx peak power & $P_{\mathrm{tx}}$ & $4 \mathrm{~kW}$ \\
Duty cycle & $d c$ & $15 \%$ \\
System temperature & $T$ & $300 \mathrm{~K}$ \\
Losses (atmospheric, system, receiver & $L \cdot F$ & $5.7 \mathrm{~dB}$ \\
noise, 2-way) & $L_{\mathrm{az}}$ & $0.2 \mathrm{~dB}$ \\
Azimuth loss & & \\
\hline \hline
\end{tabular}

enough to ensure sufficient ambiguity suppression but small enough to obtain a resolution of $5 \mathrm{~m}$.

Regarding the elevation dimension, a chirp bandwidth of $B_{\mathrm{rg}}=30 \mathrm{MHz} / \sin \left(\Theta_{\mathrm{i}}\right)$ is necessary to ensure a ground range resolution of $5 \mathrm{~m}$. Furthermore, the transmit antenna height is chosen adaptively to illuminate for all incident angles a swath of $105 \mathrm{~km}$ on ground, entailing an effective height $d_{\mathrm{ev}, \mathrm{tx}}$ varying from 0.19 to $0.31 \mathrm{~m}$ leading to a maximum antenna gain $G_{\mathrm{tx}, \max }$ from 37.2 to $39.3 \mathrm{~dB}$, respectively (cf. Fig. 7 and Table III). The height variation is achieved by an antenna consisting of multiple elements in elevation, which can be individually activated while ensuring constant transmit peak power independent of the antenna area. ${ }^{5}$ The switch between the subswaths is achieved by an electrical steering of the transmit beam where an optimum pointing of the antenna to the subswath is assumed. An interelement spacing of $2 \mathrm{~cm}$ is suitable to guarantee full coverage while avoiding grating lobes, thus requiring in total a number of 16 elements [19]. Note that, despite this spacing, the exact effective heights as derived earlier will be assumed in the following.

On receive, a large number of independent antenna elements allow for beamforming in elevation, thus ensuring a high gain and a sufficient suppression of range ambiguities. As an example, the scan-on-receive (SCORE) technique, which uses a real-time beamforming to scan the reflected pulse as it travels over the ground, can be applied [20], [21]. As a result, the overall antenna gain $G_{\mathrm{rx}}=N \cdot G_{\mathrm{rx}, j}$ can be assumed nearly independent of the incident angle $\Theta_{\mathrm{i}}$ and is simply defined by the receiving antenna area. ${ }^{6}$ Problems may occur with this technique by topography, and it might be necessary to apply a more sophisticated approach to cope with the problems arising from height variations within the scene [23], [24]. Assuming the antenna to be tilted such that it points to the center of the imaged region, the steering capability of the elevation beam requires a variation of $\pm 13^{\circ}$ with respect to antenna boresight in order to cover the 400-km swath. Within the different subswaths, the necessary scan angles to perform SCORE vary from $\pm 4.2^{\circ}$ in near range to $\pm 2.6^{\circ}$ in far range. This means that, for the near-range burst, the necessary element spacing in elevation to avoid grating lobes is $8 \mathrm{~cm}$. In consequence, the complete range requires a spacing on the order of $2 \mathrm{~cm}$, while a sufficiently high gain $\left(G_{\mathrm{rx}}=N \cdot G_{\mathrm{rx}, j}=55.3 \mathrm{~dB}\right)$ is ensured by a receiving antenna height $h_{\mathrm{ev}, \mathrm{rx}}$ of $2 \mathrm{~m}$. Finally, this results in a number of $N_{\mathrm{el}}=100$ subantenna elements. It should be noted that 100 elements are needed for the complete coverage, while within a single burst, the real-time steering of the SCORE would only require 25 elements. This can be exploited to reduce hardware complexity, e.g., by a suitable analog presumming of four channels each to "preset" the respective subswath, thus remaining only 25 presummed channels to be steered in real time.

\footnotetext{
${ }^{5}$ This can, e.g., be achieved by an analog solution enabling to switch the power feeds, thus adapting the radiated power per area. Another possibility for the "decoupling" of radiated power and effective transmit height is given by a frequency multiplex technique on transmit [25]. A detailed investigation of this issue is however beyond the scope of this paper.

${ }^{6} \mathrm{~A}$ very large antenna height might result in a very narrow footprint which no longer covers the complete pulse on ground, resulting in a gain loss for certain frequency components of the chirp. In this case, a frequency-dependent steering has to be implemented as addressed in [21] and elaborated in [22].
} 


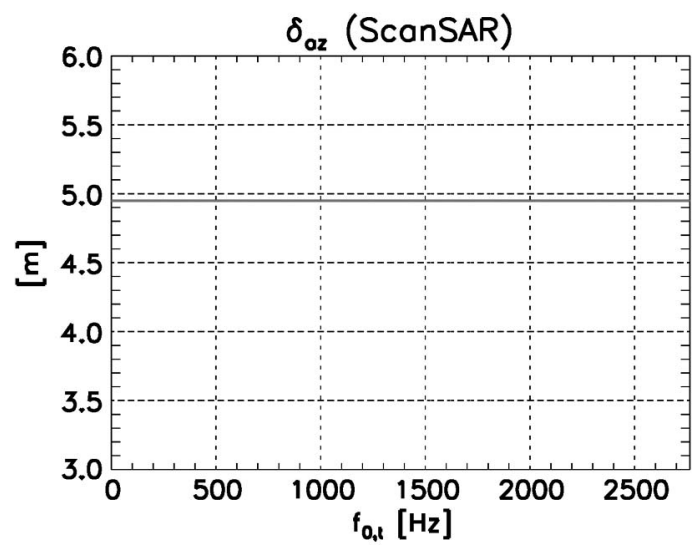

Fig. 8. Geometric resolution in azimuth $\left(\delta_{\mathrm{az}}\right)$ versus $f_{0, \mathrm{t}}$ for all subswaths.

The system is shown in Fig. 7, and all parameters are summarized in Table III. Note that Fig. 7 shows an exemplary architecture, but of course, it is also possible to, for example, employ T/R modules, thus making a separate Tx antenna unnecessary. In addition, this would allow for benefiting from the long receive aperture also on transmit, e.g., by phase tapering or by the frequency multiplex technique described in [25]. This would ease the upper limit imposed on the emitted power by a usually small Tx antenna in combination with the technologically possible power density per surface area. Furthermore, a long Tx antenna could enable novel techniques which allow for relaxing the timing constraints by continuously adapting the $\mathrm{Tx}$ phase center position as discussed in [26].

\section{B. Performance Analysis}

In the present section, a point target analysis is carried out for the previously introduced ScanSAR system, allowing for the derivation of key performance figures such as $\delta_{\mathrm{az}}, A A S R_{N}$, and NESZ. This performance analysis includes the dependence on the target position represented by the center frequency $f_{0, t}$, thus showing possible performance variations depending on the target's location within the antenna footprint. The simulated data are focused with a standard stripmap processor, where the most common approach to process only a single burst for each target is chosen. This means that, for a specific target, a subband of width $B_{\mathrm{B}}$ around the target's center frequency $f_{0, \mathrm{t}}$ is considered.

The evaluation of the geometric resolution in azimuth $\delta_{\mathrm{az}}$ according to Fig. 8 shows a constant value over the PRF and $f_{0, t}$. For all subswaths, the same resolution of $5 \mathrm{~m}$ is achieved.

Fig. 9 shows the $\operatorname{AASR}\left(A A S R_{N, \mathrm{~B}}\right)$ versus $f_{0, \mathrm{t}}$ for the different subswaths. As expected, the worst case regarding the azimuth ambiguities is encountered for the respectively maximum $f_{0, \mathrm{t}}$ (cf. Table II), i.e., targets situated at the very edge of the burst. As a result, one obtains an $A A S R_{N, \mathrm{~B}}$ better than approximately $-22 \mathrm{~dB}$.

In order to derive the NESZ of the system, signal and noise power have to be taken into account. First, the ScanSARinherent loss of the signal power in dependence on the target position is determined for each of the subswaths as shown in Fig. 10. To concentrate only on the variation, the values are normalized to the optimum at $f_{0, \mathrm{t}}=0$. The obtained "scal-

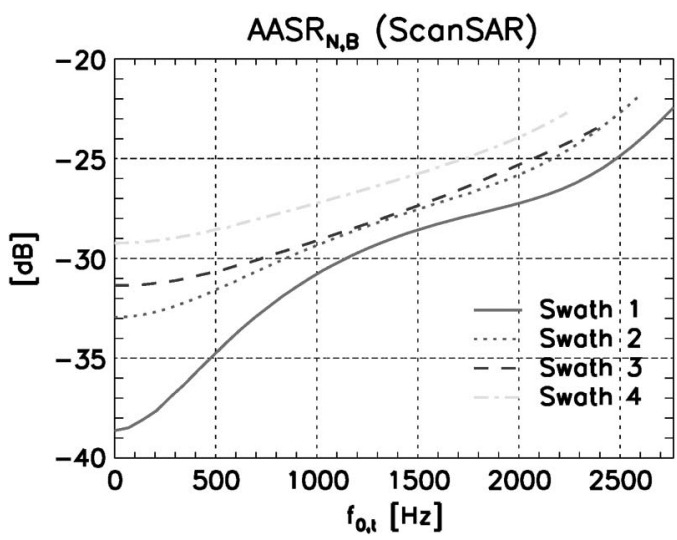

Fig. 9. Ambiguous energy suppression $\left(A A S R_{N, \mathrm{~B}}\right)$ versus $f_{0, \mathrm{t}}$ for all four subswaths.

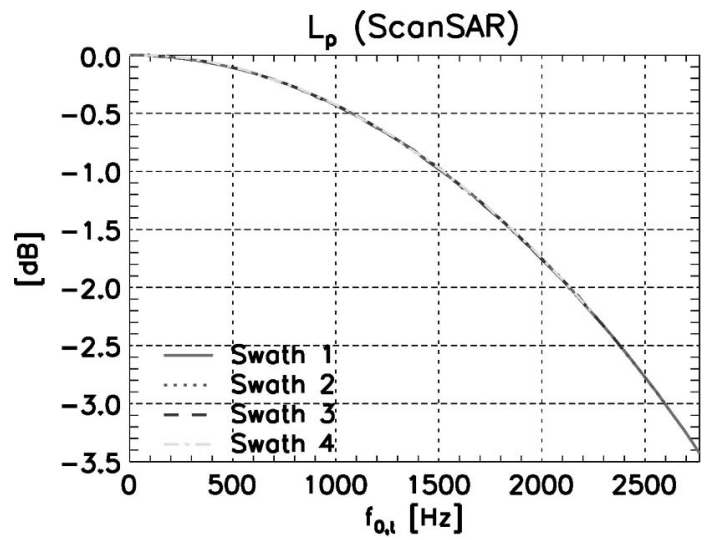

Fig. 10. Signal power loss $L_{p}$ versus $f_{0, \mathrm{t}}$ for all four subswaths. The worst case for each swath varies with the respectively maximum center Doppler frequency.

loping" has the same characteristic for each subswath, but the worst case varies according to the respective maximum center Doppler frequency listed in Table II: One obtains a maximum decrease of the signal power from $\sim 3.4 \mathrm{~dB}$ in near range (solid red line, "swath 1 ") to $\sim 2.2 \mathrm{~dB}$ in far range (dotted-dashed light blue line, "swath 4").

In addition, the noise power scaling $\Phi_{\mathrm{bf}}$ induced by the digital processing network is investigated. It is quantified by (21) and depends on the PRF of the respective subswath. Furthermore, a dependence on the target center frequency $f_{0, t}$ is observed, entailing a scallopinglike effect. Similar to the scalloping, the worst case is represented by the respectively maximum $f_{0, \mathrm{t}}$. As discussed in [2], this is caused by the fact that the scaling of ambiguous energy as well as noise power is dominated by the outermost bands of the Doppler spectrum while the amplification in the inner bands remains moderate. In the given scenario, an uncritical maximum increase of noise power by $\sim 0.2 \mathrm{~dB}$ induced by the beamforming network is obtained (cf. Fig. 11, dotted-dashed line).

Including the aforementioned results for signal and noise power scalloping and taking into account the sensor dimensions and system parameters according to Tables II and III, the NESZ can be calculated using (10). For each of the subswaths, this yields two characteristics which are shown in Fig. 12. The solid 


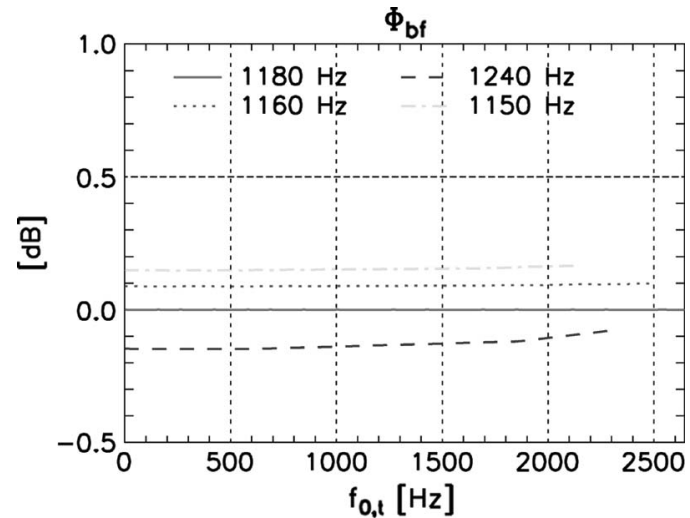

Fig. 11. SNR scaling factor of the digital processing network $\left(\Phi_{\mathrm{bf}}\right)$ versus $f_{0, t}$. Results for all subswaths taking into account the respective PRF.

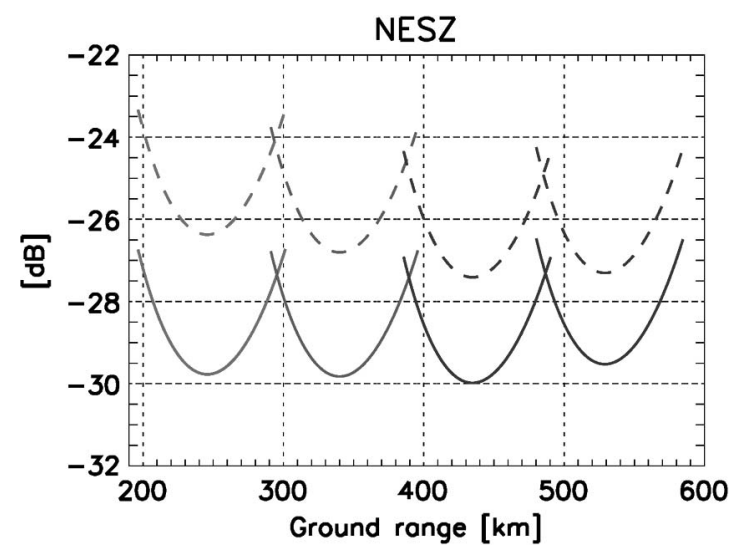

Fig. 12. NESZ versus ground range for the case where a bandwidth of $B_{\mathrm{B}}$ around a varying $f_{0, \mathrm{t}}$ is processed. The solid lines represent the best case for $f_{0, t}=0$, while the dashed lines show the worst case obtained for maximum $f_{0, \mathrm{t}}$.

lines give the best case achieved for a target centered within the antenna footprint, i.e., $f_{0, t}=0$, while the dashed lines represent the worst case which is obtained for targets located at the outermost possible position. In any case, an NESZ that is better than $-23 \mathrm{~dB}$ is achieved.

\section{Multichannel Tops Mode}

In a next step, the operation of multichannel systems in TOPS mode is investigated. Therefore, TOPS is applied to the ScanSAR system discussed earlier, and an adapted multichannel processing approach for TOPS systems is introduced. Finally, a performance analysis is carried out for a system of same coverage and resolution as in the ScanSAR operation. Regarding the subsequent analysis, it should be noted that the original system was designed for ScanSAR. Thus, the results obtained for TOPS do not represent the full possible performance but give a good indication about the potentials and challenges for the operation of multichannel systems in TOPS mode.

\section{A. Timing and System Parameters}

As mentioned, the TOPS mode is applied to the aforementioned ScanSAR system design, and consequently, the system parameters can be looked up in Table III. In addition, the TOPS azimuth steering coefficient $\alpha$ has to be chosen, and some minor adaptations have to be done, which will be derived in the following. First, the dwell time $T_{\mathrm{D}, i}$ represents the integration time which is necessary to ensure the required azimuth resolution. A first estimate for $T_{\mathrm{D}, i}$ can be derived using (11), and for the given antenna dimensions and parameters, it is chosen to yield a Doppler bandwidth of $1.32 \mathrm{kHz}$, which is slightly above the value for the ScanSAR system. The corresponding values for each subswath are listed in Table IV. Next, similar to ScanSAR, the timing of TOPS has to ensure continuous coverage, which is expressed as follows, where the cycle time $T_{\mathrm{C}}$ is defined by the sum over all burst times $T_{\mathrm{B}, \mathrm{TOPS}, i}$ :

$$
T_{\mathrm{B}, \mathrm{TOPS}, i} \geq \frac{T_{\mathrm{C}}-\alpha \cdot T_{\mathrm{D}, i}}{\alpha} .
$$

Equation (23) offers "some" degree of freedom, as $T_{\mathrm{B}, \mathrm{TOPS}, i}$ as well as $\alpha$ can be varied. In order to prevent a too high squint angle resulting from a too long burst, $\alpha=5$ is chosen. Solving the resulting set of equations leads for each subswath to the TOPS burst time and corresponding maximum steering angle $\Theta_{\mathrm{az}, \mathrm{max}, i}$ as summarized in Table IV. The respective maximum target center frequencies are calculated according to

$$
f_{0, t, \max }=\frac{2 \cdot v_{\mathrm{s}}}{\lambda} \cdot \sin \left(\frac{v_{\mathrm{g}} \cdot(\alpha-1)}{2 \cdot R_{0, i}} \cdot\left(T_{\mathrm{B}, \mathrm{TOPS}, i}-T_{\mathrm{D}, i}\right)\right) .
$$

\section{B. Adaptively Squinted Multichannel Processing for TOPS}

In TOPS, the instantaneous Doppler spectrum is defined by the antenna pattern, but steering with the aforementioned parameters leads to squint angles of up to $\pm 1.69^{\circ}$. Thus, while the received bandwidth at each instant of time is sampled according to Nyquist, the overall Doppler bandwidth $B_{\text {TOPS }}$ clearly exceeds the azimuth system bandwidth of $N \cdot P R F$ (cf. Fig. 13, left). As a result, conventional reconstruction of a Doppler band around $f=0$ cannot be applied anymore, but a time-dependent approach is necessary to perform the multichannel reconstruction. The basic principle is sketched on the right part of Fig. 13, showing the idea of exploiting the dependence of squint angle versus time by adapting the respective center frequency of the reconstructed band $f_{0}(t)$ to the actual center frequency of the TOPS steering ("ramping").

In the following, a step size of PRF is chosen to adapt the center frequency of the processing filters. Furthermore, processing is performed in the Doppler domain, which requires a minimum signal length of $T_{\mathrm{D}}$ to consider the complete echo of a specific target. This leads to a blockwise processing approach, whose main steps might look as follows (and whose detailed derivation is not within the scope of this paper).

1) Starting with the minimum target center frequency, the closest integer multiple of PRF is determined, leading to a block of $f_{0, \mathrm{t}}$, where $\left|f_{0, \mathrm{t}}-k \cdot P R F\right|<P R F / 2, k$ integer, holds true.

2) The block length in the time domain is chosen such that the full echo of each of the above $f_{0, t}$ is considered.

3) Reconstruction and focusing around $k \cdot P R F$ are performed, and only results obtained for the actual $f_{0, t}$ are 
TABLE IV

TOPS PARAMETERS OF DiFFERENT SUBSWATHS

\begin{tabular}{lccccc}
\hline \hline \multicolumn{1}{c}{ Parameter } & Symbol & \multicolumn{4}{c}{ Value } \\
& & Subswath 1 & Subswath 2 & Subswath 3 & Subswath 4 \\
\hline TOPS burst duration & $T_{\mathrm{B}, \mathrm{TOPS}}$ & $1.47 \mathrm{~s}$ & $1.49 \mathrm{~s}$ & $1.51 \mathrm{~s}$ & $1.54 \mathrm{~s}$ \\
Dwell time & $T_{\mathrm{D}, i}$ & $0.271 \mathrm{~s}$ & $0.288 \mathrm{~s}$ & $0.310 \mathrm{~s}$ & $0.334 \mathrm{~s}$ \\
Azimuth angle range & $\Theta_{\mathrm{az}, \max }$ & $\pm 1.70^{\circ}$ & $\pm 1.62^{\circ}$ & $\pm 1.52^{\circ}$ & $\pm 1.43^{\circ}$ \\
Max.center Doppler & $f_{0, \mathrm{t}, \max }$ & $11.76 \mathrm{kHz}$ & $11.05 \mathrm{kHz}$ & $10.27 \mathrm{kHz}$ & $9.50 \mathrm{kHz}$ \\
\hline \hline
\end{tabular}

TOPS Time-Frequency Characteristic

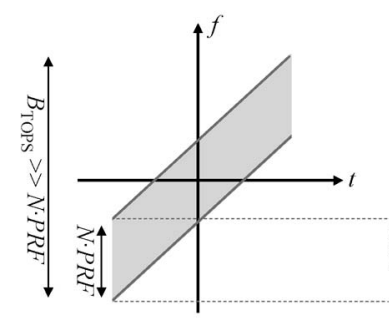

Squinted Multi-Channel

Processing Spectrum

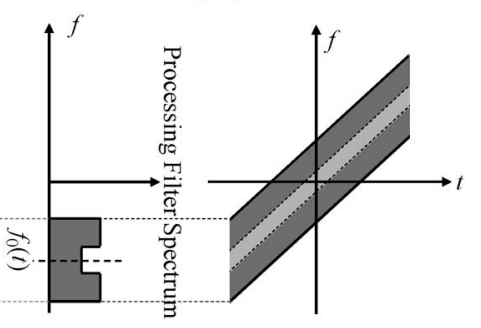

Fig. 13. (Left) TOPS time-frequency characteristic. (Right) Idea of adapting the Doppler spectrum of multichannel processing to TOPS. In the optimum case, the reconstructed band is centered adaptively around a varying frequency $f_{0}(t)$ to ideally cover the TOPS spectrum.

\section{"Staircase" Multi-Channel Processing}

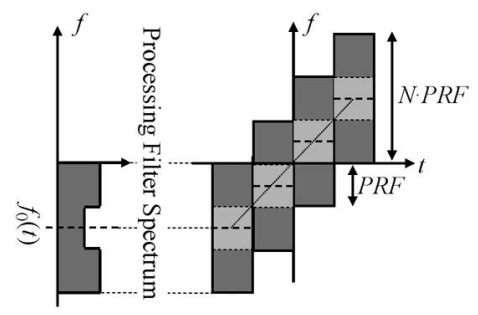

Fig. 14. Applied squinted multichannel processing. The reconstructed bandwidth is kept constant and centered adaptively in a staircase-like manner on the target center frequency given by the diagonal dotted line.

considered, meaning that the results obtained for echoes which are only partly in the considered block are omitted.

This scheme is continued until the maximum target center frequency is reached, yielding the "staircase-like" behavior as shown in Fig. 14. Note that, for reasons of clarity, the overlap in time of adjacent blocks is not depicted.

The resulting output signal of this "staircase" reconstruction is still ramped but free of aliasing. Consequently, further processing as suggested in [6], [18], [27], or [28] can now be applied.

\section{Performance Analysis}

In the following, the key performance figures $\delta_{\mathrm{az}}, A A S R_{N}$, and $N E S Z$ of the TOPS mode are determined by a pointtarget analysis considering the "staircase-like" adaptation of the reconstruction as introduced earlier. The simulations are carried out for a single element size of $2.5 \mathrm{~cm}$, leading to a nearly ideally steered pattern. If the element size becomes too big, the single-element azimuth pattern, as well as grating lobes, can influence the performance.

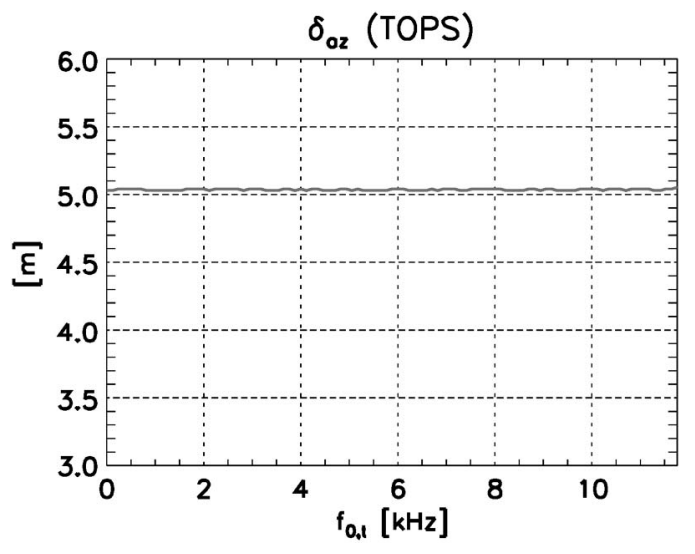

Fig. 15. Geometric resolution in azimuth $\left(\delta_{\mathrm{az}}\right)$ versus $f_{0, \mathrm{t}}$ for TOPS mode operation.

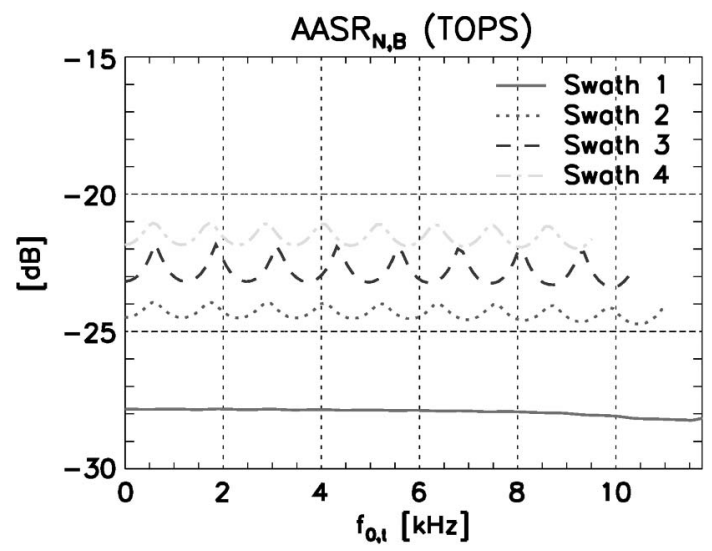

Fig. 16. Azimuth ambiguous energy suppression $\left(A A S R_{N, \mathrm{~B}}\right)$ versus $f_{0, \mathrm{t}}$ for TOPS mode operation. The different line styles represent the results obtained for the four different subswaths.

As can be seen from Fig. 15, the TOPS mode yields the same azimuth resolution $\delta_{\mathrm{az}}$ of $\sim 5 \mathrm{~m}$ as in ScanSAR but requires a slightly increased bandwidth of $1.32 \mathrm{kHz}$ due to the effectively shrunk pattern of TOPS. As the single-element characteristic is assumed constant, the resolution in TOPS is constant, independent of the target position.

Next, the suppression of azimuth ambiguous energy $\left(A A S R_{N, \mathrm{~B}}\right)$ by TOPS is shown in Fig. 16 for all four subswaths. The $A A S R_{N, \mathrm{~B}}$ varies with the offset of the target center frequency $f_{0, \mathrm{t}}$ from the respective center frequency of the reconstructed band. As this center frequency is switched with integer multiples of PRF, the $A A S R_{N, \mathrm{~B}}$ shows only a variation over an interval of width $\mathrm{PRF}$ which is periodically continued. As the beamsteering ensures the same pattern independent of the target position, the different performance is 


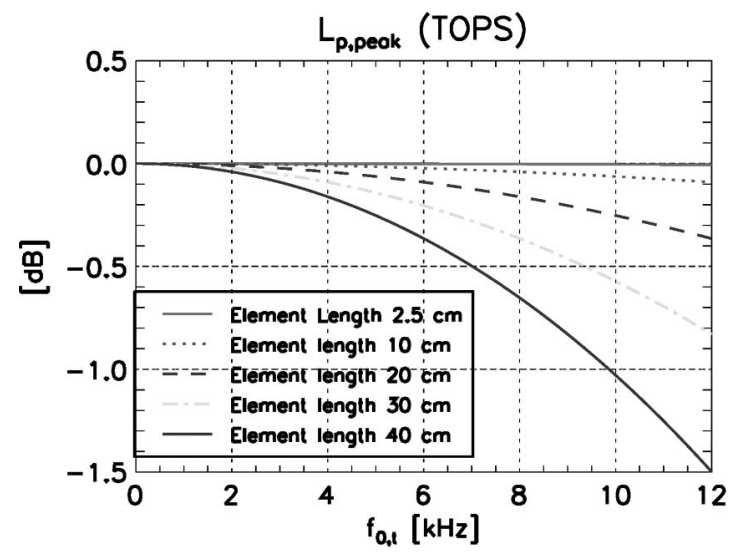

Fig. 17. Peak power loss normalized to optimum value at $f_{0, \mathrm{t}}=0\left(L_{p}\right)$ versus $f_{0, t}$ for TOPS mode operation for antenna element sizes of $2.5,10,20$, 30 , and $40 \mathrm{~cm}$. The larger the single element, the higher the peak power loss.

only caused by the respective multichannel processing of the signal, which is governed by the different PRF values of the respective swaths. Hence, in contrast to ScanSAR, the influence of the antenna pattern is eliminated. This can be seen from the solid line in Fig. 16 which corresponds to the optimum PRF and shows a nearly constant value for all target positions as the sampling is uniform in this case and no multichannel processing is applied. The minor offset from the constant value for high target center frequencies is possibly caused by the large corresponding squint angle. In reverse, the other characteristics in Fig. 16 (dotted, dashed, and dotted-dashed) show the scalloping introduced by the multichannel processing for the respective PRF. The results show a suppression of better than $-21 \mathrm{~dB}$, nearly identical to the ScanSAR results.

In order to derive the NESZ of the system, first, the loss of signal peak power has to be determined. Only for an optimum TOPS pattern that every target is illuminated by the same antenna pattern and no scalloping is encountered. In reality, the single-element characteristic of the array antenna has to be considered, causing a small scalloping effect which is directly given by the characteristic drop-off with Doppler frequency. Fig. 17 shows this effect in dependence on the target center frequency for different element sizes. One recognizes that the assumed element size of $2.5 \mathrm{~cm}$ leads to a nearly optimum behavior close to $0 \mathrm{~dB}$. However, even for an antenna element size of $40 \mathrm{~cm}$, TOPS yields a clearly better scalloping result than ScanSAR.

In addition to the peak power, the effect of the effectively shrunk pattern on the signal power has to be considered. This effect can be quantified by the azimuth loss factor $L_{\mathrm{az}}$, and one obtains a value of $-1.40 \mathrm{~dB}$. Furthermore, the noise scaling introduced by the digital processing network does not depend on the acquired signal but only on the processing. Consequently, for the same set of parameters, this scalloping is the same for both modes. As the processed Doppler bandwidth has been only slightly increased and, furthermore, the impact of the processing network is nearly negligible, Fig. 11 not only is exact for ScanSAR but also can be considered as a valid approximation for TOPS. Finally, the NESZ for the TOPS mode can be approximated by the optimum value for ScanSAR, which is represented by the solid line in Fig. 12, and taking into account the increased azimuth loss of $1.40 \mathrm{~dB}$ compared to a value of $0.1 \mathrm{~dB}$ for ScanSAR. In consequence, TOPS mode ensures for any target an NESZ better than $\sim-25 \mathrm{~dB}$.

\section{TOPS Versus ScanSAR}

The results obtained for TOPS as well as for ScanSAR show the same performance with respect to coverage and resolution and highlight directly the big advantage of TOPS: The steering of the azimuth antenna footprint during signal acquisition ensures a constant signal power independent on the target position, which allows for removing or clearly reducing the scalloping. In particular, with regard to the NESZ, the large scalloping in ScanSAR of up to $3.4 \mathrm{~dB}$ has been completely removed. Furthermore, longer bursts are possible with TOPS, reducing the losses when switching between different subswaths. These advantages come at the cost of additional hardware, which are necessary to enable the azimuth beamsteering in TOPS, and an increased computational complexity, mainly caused by the blockwise processing.

Keeping in mind that the azimuth antenna dimensions were designed for ScanSAR, a system optimized for TOPS would offer further potential to fully exploit the TOPS mode. In particular, a careful adjustment of steering coefficient and antenna patterns could, for example, reduce the rather large azimuth loss factor.

\section{DISCUSSION}

Multichannel SAR systems operating in stripmap mode were shown to be unsuitable for the mapping of ultrawide swaths of several hundred kilometers as this would require antennas with an unreasonable length. Thus, this paper presented, as a possible solution, the operation of multichannel SAR systems in burst modes, such as ScanSAR and TOPS. In a first step, the application of multichannel processing algorithms to burst modes was analyzed theoretically, investigating the Dopplerfrequency-dependent multichannel processing with respect to the particular properties of burst-mode data. As the target position determines the spectral band of its echo, different targets are processed with different subbands of the processing filter's colored spectrum. This results in a variation of the performance depending on the target position, introducing a scalloping-like effect both for TOPS and ScanSAR. This effect was derived analytically when extending the expressions for performance estimation to burst modes. Next, a multichannel ScanSAR system was designed which demonstrated the possibility to image a swath of $400 \mathrm{~km}$ with a resolution of $5 \mathrm{~m}$. Furthermore, performance analyses illustrated the introduced scalloping of residual azimuth ambiguities and the SNR scaling, thus verifying the theory. Additionally, the designed system was also operated in TOPS mode, and an innovative approach for processing multichannel TOPS data was presented: By adaptively "ramping" the processing filter functions, the multichannel reconstruction was adapted to the "squinted" time-frequency characteristic of TOPS. In the end, TOPS mode showed a similar performance as ScanSAR with respect to coverage, geometric resolution, and 
azimuth ambiguity suppression. The big advantage of TOPS is given by the reduced scalloping, in particular with respect to the NESZ. One should keep in mind that the system design was optimized to ScanSAR, meaning that there is further potential to optimized TOPS performance by a better adaptation of the system design to this mode.

In conclusion, the results for both modes show that this new class of multichannel systems enables frequent mapping on a global scale with unprecedented detail. This means that such systems open up an entirely new field of SAR operation and introduce a new degree of freedom in SAR system design, e.g., with respect to the antenna dimensions. Consequently, multichannel burst modes represent an important aspect in the "toolkit" for advanced SAR sensors which are capable of answering the needs of future remote sensing missions.

As a next step, based on the analysis of multichannel burstmode operation, focus should be on the adaptation of the system design to multichannel TOPS operation in combination with a further development of the TOPS multichannel processing. In this context, more sophisticated burst modes allowing for even higher resolutions and swath widths deserve special interest [7]. Another promising concept is the application of burst modes to large reflector antennas with a feed array enabling digital signal processing both in azimuth and range dimensions. The large reflector dimensions ensure a high antenna gain in combination with a narrow beam whose steering angle-and consequently the illuminated footprint on ground-is directly linked to a specific feed element (or a subgroup of them). This makes such systems ideally suited for multibeam applications, be it to enable effective range ambiguity suppression or to image multiple elevation beams simultaneously [23], [29], [30].

\section{APPENDIX}

\section{A. Multichannel Range Cell Migration}

The range history of a pointlike target at distance $R_{0}$ migrates over several range resolution cells, thus spanning a range from $R_{0}$ to $R_{0}+\Delta R$. In addition, in multichannel systems based on a single Tx antenna, the same target is "seen" under different minimum slant-range distances and with varying range curvatures, depending on the respective distance to the transmit aperture. As illustrated hereinafter, this might result in a different range cell migration for each receive aperture (Fig. 18).

The unambiguous azimuth signal is obtained by separately reconstructing each range bin, i.e., each line of the data array. Hence, it has to be ensured that the contributions from a specific target in the range-Doppler domain are coregistered to the same "reference" range bin in all receivers' signal memory spaces. In this regard, first, a large receiver spacing might result in a large relative range offset $\Delta R_{0, j}$, which exceeds the range resolution, thus requiring a range shift to correct for. Second, the differential range curvature $\Delta R_{\mathrm{curv}, j}$ might also be in the order of a range resolution cell. As a consequence, the echo of a specific target is stored in different range bins according to the respective receiver's position, thus precluding correct reconstruction. It should be noted that $\Delta R_{0, j}$ and $\Delta R_{\mathrm{curv}, j}$ consider the two-way path difference, as this is relevant for the different delay times.

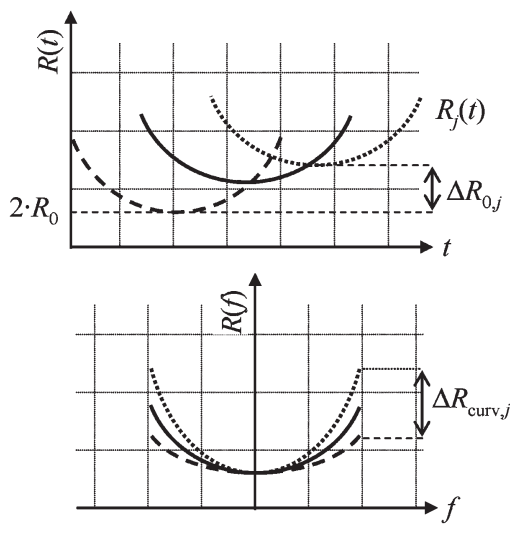

Fig. 18. Multichannel range cell migration. Depending on the receiver position, the same target shows different range histories. (Top) Relative twoway slant-range offset $\Delta R_{0, j}$ for three receivers. (Bottom) Varying curvature versus Doppler frequency, resulting in an offset $\Delta R_{\mathrm{curv}, j}$ (two-way, each curve normalized to its respective $R_{0}$ ).

The variation in slant range can be directly derived from the effective phase center definition, which includes - in quadratic phase approximation - the addition of a constant phase. This phase originates from the previously discussed differences regarding the minimum slant range of the range history, leading to the following two-way offsets according to the spacing $\Delta x_{j}$ of the respective receiver $j$ from the transmitter:

$$
\Delta R_{0, j}=\frac{v_{\mathrm{g}}}{v_{\mathrm{s}}} \cdot \frac{\Delta x_{j}^{2}}{4 \cdot R_{0}} .
$$

In order to evaluate the impact of the different range cell migrations, one single-platform system and one distributed constellation are analyzed for L-band as well as X-band and a slant range of $735 \mathrm{~km}$. In this context, the maximum receiver separation describes the largest distance with respect to a reference receiver at the transmitter position. As the range curvature depends also on the illumination time, a value is chosen which ensures an azimuth resolution on the order of $1 \mathrm{~m}$. Furthermore, for the following considerations, a range resolution in the same order is assumed. The obtained results are summarized in the subsequent table which also contains the reconstruction phase errors from the analysis in the following paragraph.

Regarding the differential range curvatures, Table $\mathrm{V}$ shows for both systems and bands an error far below the range resolution, and consequently, this effect can be neglected. This is good news, as the correction of the curvature could only be carried out in the Doppler frequency domain. Hence, on the one hand, the full-i.e., unaliased-Doppler spectrum would be required, but on the other hand, a correction would be necessary prior to reconstruction, which is a contradiction in terms. Concerning the relative range offset, a negligible value is encountered in single-platform systems, but a sparse array constellation might require compensation. For each channel, the respective offset is a constant value independent on the Doppler frequency but with slight variation in dependence of the slant range according to (25). As the differential curvature is minor, this means that all range histories obtained at a certain $R_{0}$ can be aligned correctly by a constant shift of the received pulse in the range time domain. As a pixelwise shift cannot ensure correctly aligned range histories over the complete range 
TABLE V

Range Migration in Multichannel SAR and Resulting Reconstruction Filter Mismatch

\begin{tabular}{lcccc}
\hline \multicolumn{1}{c}{ Parameter } & $\begin{array}{c}\text { Single-platform System } \\
\text { X-Band }\end{array}$ & L-Band & $\begin{array}{c}\text { Multi-platform System } \\
\text { X-Band }\end{array}$ & L-Band \\
\hline Maximum receiver separation & \multicolumn{2}{c}{$10 \mathrm{~m}$} & $500 \mathrm{~m}$ \\
Differential range curvature $\Delta R_{\text {curv }, j}(2-$ way $)$ & $<10^{-8} \mathrm{~m}$ & $<10^{-6} \mathrm{~m}$ & $<10^{-5} \mathrm{~m}$ & $<10^{-3} \mathrm{~m}$ \\
Relative range offset $\Delta R_{0, j}(2$-way) & $<10^{-4} \mathrm{~m}$ & $<0.1 \mathrm{~m}$ \\
Maximum Range Migration $\Delta R_{j}$ & $<50 \mathrm{~m}$ & $<2700 \mathrm{~m}$ & $<50 \mathrm{~m}$ & $<2700 \mathrm{~m}$ \\
$\Delta \varphi_{\tau, j}$ (uncompressed, $\left.\tau=10 \mu \mathrm{s}\right)$ & $<10^{-2}$ & $<10^{-3 \circ}$ & $<5^{\circ}$ & $<1.5^{\circ}$ \\
$\Delta \varphi_{j}$ (range compressed) & $<10^{-4}$ & $<10^{-3 \circ}$ & $<0.1^{\circ}$ & $<0.6^{\circ}$ \\
\hline \hline
\end{tabular}

migration, the alignment has to be performed by a phase ramp in the range frequency domain, yielding a time shift according to $\Delta R_{0, j}$. This leaves the azimuth phase unchanged, thus not affecting the following azimuth processing steps. For example, the shift can be directly incorporated in the range compression by using an appropriately shifted replica, which has to be adapted to the respective $R_{0}$ to ensure exact solution. In a brute force approach, this would mean that, for each slant range, the compression is performed separately. As this entails huge computational load and as the variation of $\Delta R_{0, j}$ over $R_{0}$ is slow, one could think of a range compression in blocks. An investigation of optimized correction algorithms is beyond the scope of this paper as the focus of the research is directed to single-platform systems.

\section{B. Reconstruction Filter Mismatch}

In a second step, the reconstruction filter functions $P_{j}(f)$ are taken into account, as they depend on $R_{0}$. As indicated by (1) and (2), the system matrix $\mathbf{H}$ can be decomposed into a matrix $\mathbf{H}^{\prime}$ multiplied by a diagonal matrix $\mathbf{D}$ whose elements at position $(j, j)$ represent the dependence on $R_{0}$

$$
(\mathbf{D})_{j, j}=\exp \left[-\mathrm{j} \cdot \frac{v_{\mathrm{g}}}{v_{\mathrm{s}}} \cdot \frac{\pi \cdot \Delta x_{j}^{2}}{2 \cdot \lambda \cdot R_{0}}\right] \text {. }
$$

Thus, according to basic matrix algebra, the matrix $\mathbf{P}$ is made up of the inverse from $\mathbf{H}^{\prime}$ multiplied by $\mathbf{D}^{-\mathbf{1}}$ which simply consists of the inverse diagonal elements. As a result, the dependence of each channel $j$ on $R_{0}$ is characterized as follows:

$$
P_{j}(f) \propto \exp \left[\mathrm{j} \cdot \frac{v_{\mathrm{g}}}{v_{\mathrm{s}}} \cdot \frac{\pi \cdot \Delta x_{j}^{2}}{2 \cdot \lambda \cdot R_{0}}\right] .
$$

As the range history spans several range bins, a signal obtained for a target at $R_{0}$ will be partly reconstructed by filters calculated for $R_{0}+\Delta R_{j}$. Thus, range migration introduces a phase mismatch $\Delta \varphi_{j}$ between the signal and the reconstruction. As differential range curvature can be neglected and the relative range offset is assumed to be corrected, $\Delta \varphi_{j}$ is only determined by the maximum range migration $\Delta R_{j}$ of channel $j$. Note that, in this context, the one-way slant range is relevant, since the Tx path is the same for all channels.

$$
\begin{aligned}
\Delta \varphi_{j} & =\frac{v_{\mathrm{g}}}{v_{\mathrm{s}}} \cdot \frac{\pi \cdot \Delta x_{j}^{2}}{2 \cdot \lambda}\left(\frac{1}{R_{0}}-\frac{1}{R_{0}+\Delta R_{j}}\right) \\
& =\frac{v_{\mathrm{g}}}{v_{\mathrm{s}}} \cdot \frac{\pi \cdot \Delta x_{j}^{2}}{2 \cdot \lambda}\left(\frac{\Delta R_{j}}{R_{0} \cdot\left(R_{0}+\Delta R_{j}\right)}\right) .
\end{aligned}
$$

This result enables to convert the range migration in Table $\mathrm{V}$ in respective phase errors, where $\Delta \varphi_{j}$ refers to the compressed pulse while $\Delta \varphi_{\tau, j}$ considers the uncompressed pulse. Here, a pulse length $\tau=10 \mu \mathrm{s}$ is assumed, and correspondingly, the maximum range mismatch is increased by $\tau \cdot \mathrm{c}_{0}=3 \mathrm{~km}$, with the speed of light $c_{0}$. Both possibilities are analyzed, as in principle, the aliased data of each channel can be range compressed either before reconstruction or afterward. Nevertheless, the obtained values (cf. Table V) allow for the conclusion that the phase errors are negligible for range-compressed data but have to be considered for uncompressed data gathered by multiplatform systems with large receiver separations. Consequently, from the reconstruction point of view, range compression prior to reconstruction is favorable, as the extension of the target echo is reduced by the pulse length and the effective $\Delta R$ will be smaller.

\section{REFERENCES}

[1] G. Krieger, N. Gebert, and A. Moreira, "Unambiguous SAR signal reconstruction from nonuniform displaced phase center sampling," IEEE Geosci. Remote Sens. Lett., vol. 1, no. 4, pp. 260-264, Oct. 2004.

[2] N. Gebert, G. Krieger, and A. Moreira, "Digital beamforming on receive: Techniques and optimization strategies for high-resolution wideswath SAR imaging," IEEE Trans. Aerosp. Electron. Syst., vol. 54, no. 2, pp. 564-592, Apr. 2009

[3] N. Gebert, G. Krieger, and A. Moreira, "Optimization strategies for multiaperture SAR imaging with high performance," in Proc. IRS, Cologne, Germany, 2007.

[4] K. Tomiyasu, "Conceptual performance of a satellite borne, wide swath synthetic aperture radar," IEEE Trans. Geosci. Remote Sens., vol. GRS-19, no. 2, pp. 108-116, Apr. 1981.

[5] R. K. Moore, J. P. Claassen, and Y. H. Lin, "A scanning spaceborne synthetic aperture radar with integrated radiometer," IEEE Trans. Aerosp. Electron. Syst., vol. AES-17, no. 3, pp. 410-421, May 1981.

[6] F. De Zan and A. M. Monti Guarnieri, "TOPSAR: Terrain observation by progressive scans," IEEE Trans. Geosci. Remote Sens., vol. 44, no. 9, pp. 2352-2360, Sep. 2006.

[7] G. Krieger, N. Gebert, M. Younis, F. Bordoni, A. Patyuchenko, and A. Moreira, "Advanced concepts for ultra-wide-swath SAR imaging with high azimuth resolution," in Proc. EUSAR, Friedrichshafen, Germany, 2008.

[8] N. Gebert, G. Krieger, and A. Moreira, "Multi-channel ScanSAR for highresolution ultra-wide-swath imaging," in Proc. EUSAR, Friedrichshafen, Germany, 2008.

[9] N. Gebert, G. Krieger, M. Younis, F. Bordoni, and A. Moreira, "Ultra wide swath imaging with multi-channel ScanSAR," in Proc. IEEE IGARSS, Boston, MA, Jul. 2008, pp. V-21-V-24.

[10] M. Younis, F. Bordoni, N. Gebert, and G. Krieger, "Smart multi-channel radar techniques for spaceborne remote sensing," in Proc. IEEE IGARSS, Boston, MA, Jul. 2008, pp. III-278-III-281.

[11] A. Papoulis, "Generalized sampling expansion," IEEE Trans. Circuits Syst., vol. CAS-24, no. 11, pp. 652-654, Nov. 1977.

[12] J. Brown, "Multi-channel sampling of low-pass signals," IEEE Trans. Circuits Syst., vol. CAS-28, no. 2, pp. 101-106, Feb. 1981.

[13] G. Krieger, N. Gebert, and A. Moreira, "SAR signal reconstruction from non-uniform displaced phase center sampling," in Proc. IEEE IGARSS, Anchorage, AK, Sep. 2004, pp. 1763-1766. 
[14] J. Curlander and R. McDonough, Synthetic Aperture Radar-Systems and Signal Processing. New York: Wiley, 1991.

[15] R. K. Raney, "SNR in SAR," in Proc. IEEE IGARSS, Amherst, MA, Oct. 1985, pp. 994-999.

[16] R. K. Raney, "SNR considerations for an orbital SAR," in Proc. IEEE IGARSS, Washington, DC, May 1990, p. 1125.

[17] G. Krieger, A. Moreira, H. Fiedler, I. Hajnsek, M. Werner, M. Younis, and M. Zink, "TanDEM-X: A satellite formation for high-resolution SAR interferometry," IEEE Trans. Geosci. Remote Sens., vol. 45, pt. 1, no. 11, pp. 3317-3341, Nov. 2007.

[18] P. Prats, A. Meta, R. Scheiber, J. Mittermayer, J. Sanz-Marcos, and A. Moreira, "A TOPSAR processing algorithm based on extended chirp scaling: Evaluation with TerraSAR-X data," in Proc. EUSAR, Friedrichshafen, Germany, 2008.

[19] C. Fischer, C. Heer, G. Krieger, and R. Werninghaus, "A high resolution wide swath SAR," in Proc. EUSAR, Dresden, Germany, 2006.

[20] M. Suess and W. Wiesbeck, "Side-looking synthetic aperture system," Eur. Patent EP 1241487 A 1, Feb. 8, 2006.

[21] M. Suess, B. Grafmüller, and R. Zahn, "A novel high resolution, wide swath SAR system," in Proc. IEEE IGARSS, Sydney, Australia, 2001, pp. 1013-1015.

[22] C. Schaefer, Active Receive-Only Digital Frontend for Radar and SAR, Friedrichshafen, Germany, Technical Note TN-DFE-1100-AED/01, ESA contract 19648/05/NL/JA: EADS Astrium 2008.

[23] G. Krieger, N. Gebert, and A. Moreira, "Digital beamforming techniques for spaceborne radar remote sensing," in Proc. EUSAR, Dresden, Germany, 2006.

[24] G. Krieger, N. Gebert, and A. Moreira, "Multidimensional waveform encoding: A new digital beamforming technique for synthetic aperture radar remote sensing," IEEE Trans. Geosci. Remote Sens., vol. 46, no. 1, pp. 31-46, Jan. 2008.

[25] B. Grafmüller and C. Schaefer, "Hochauflösende Synthetik-AperturRadarvorrichtung und Antenne für eine hochauflösende SynthetikApertur-Radarvorrichtung," German Patent No. DE 102005062 031.0, Jun. 28, 2007.

[26] N. Gebert and G. Krieger, "Phase center adaptation on transmit for highresolution wide-swath SAR imaging," IEEE Geosci. Remote Sens. Lett., vol. 6, no. 4, pp. 782-786, Oct. 2009.

[27] P. Prats, R. Scheiber, J. Mittermayer, A. Meta, A. Moreira, and J. Sanz-Marcos, "A SAR processing algorithm for TOPS imaging mode based on extended chirp scaling," in Proc. IEEE IGARSS, Barcelona, Spain, 2007, pp. 148-151.

[28] P. Prats, R. Scheiber, J. Mittermayer, A. Meta, and A. Moreira, "Processing of sliding spotlight and TOPS SAR data using baseband azimuth scaling," IEEE Trans. Geosci. Remote Sens., vol. 48, no. 2, pp. 770-780, Feb. 2010.

[29] M. Younis, S. Huber, A. Patyuchenko, F. Bordoni, and G. Krieger, "Performance comparison of reflector- and planar-antenna based digital beamforming SAR,” Int. J. Antennas Propag., vol. 2009, p. 614 931, 2009.

[30] G. Krieger, I. Hajnsek, K. Papathanassiou, M. Eineder, M. Younis, F. De Zan, P. Prats, S. Huber, M. Werner, H. Fiedler, A. Freeman, P. Rosen, S. Hensley, W. Johnson, L. Veilleux, B. Grafmueller, R. Werninghaus, R. Bamler, and A. Moreira, "The tandem-L mission proposal: Monitoring Earth's dynamics with high resolution SAR interferometry," in Proc. IEEE RadarCon, Pasadena, CA, May 2009, pp. 1-6.

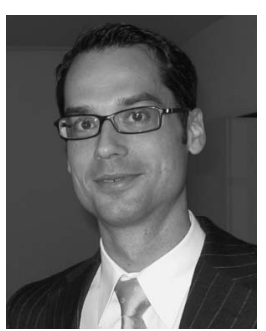

Nicolas Gebert received the Dipl.-Ing. (M.S.) degree from the Munich University of Technology (TU München), Munich, Germany, in 2003 and the Dr.-Ing. (Ph.D.) degree (with distinction) from the University of Karlsruhe (TH), Karlsruhe, Germany, in 2009.

Since 2003, he has been a Research Engineer with the Microwaves and Radar Institute (HR), German Aerospace Center (DLR), Weßling, Germany. His major research interests are innovative concepts for spaceborne synthetic aperture radar (SAR) systems for remote sensing of the Earth with high resolution and wide-swath coverage. In this area, he focuses on the development of novel digital signal processing techniques (digital beam-forming) within the frame of an integrated multichannel SAR system design. Currently, he is researching and developing innovative concepts for ultrawide-swath imaging with SAR systems based on planar arrays as well as reflector antennas.

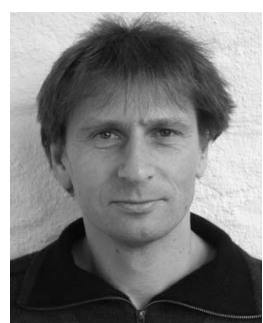

Gerhard Krieger (A'04-SM'04) received the Dipl.-Ing. (M.S.) and Dr.-Ing. (Ph.D.) degrees (with honors) in electrical and communication engineering from the Technical University of Munich, Munich, Germany, in 1992 and 1999, respectively.

From 1992 to 1999, he was with LudwigMaximilians University, Munich, where he conducted multidisciplinary research on neuronal modeling and nonlinear information processing in biological and technical vision systems. In 1999, he joined the Microwaves and Radar Institute (HR), German Aerospace Center (DLR), Weßling, Germany, where he developed signal and image processing algorithms for a novel forward-looking radar system employing digital beamforming on receive. From 2001 to 2007, he led the New SAR Missions Group which pioneered the development of advanced bistatic and multistatic radar systems as exemplified by the forthcoming TanDEM-X mission as well as innovative multichannel SAR techniques and algorithms for high-resolution wide-swath SAR imaging. Since 2008, he has been the Head of the new Radar Concepts Department, HR. He is the author of 30 peer-reviewed journal papers, four invited book chapters, more than 100 conference papers, and four patents. His current research interests focus on the development of multichannel radar techniques and algorithms for innovative multiple-input-multiple-output SAR systems, the demonstration of novel interferometric and tomographic Earth observation applications, and the conceptual design of advanced bi- and multistatic radar missions.

Dr. Krieger has received several national and international awards, including the IEEE Transactions Prize Paper Award of the Geoscience and Remote Sensing Society and the Otto Lilienthal Sabbatical of the DLR.

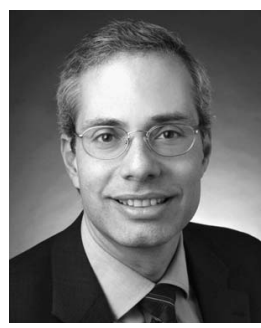

Alberto Moreira (M'92-S'96-F'04) was born in São José dos Campos, Brazil, in 1962. He received the B.S.E.E. and M.S.E.E. degrees from the Aeronautical Technological Institute (ITA), São José dos Campos, in 1984 and 1986, respectively, and the Dr. Eng. degree (with honors) from the Technical University of Munich, Munich, Germany, in 1993.

From 1996 to 2001, he was the Chief Scientist and Engineer with the SAR Technology Department, German Aerospace Center (DLR), Weßling, Germany. Since 2001, he has been the Director of the Microwaves and Radar Institute, DLR. The institute contributes to several scientific programs and space projects for actual and future airborne and spaceborne SAR missions like TerraSAR-X, TanDEM-X, and Sentinel-1. Since 2003, he has been a Full Professor in the field of microwave remote sensing with the University of Karlsruhe, Karlsruhe, Germany. In 2006, the mission proposal TanDEM-X led by his institute was approved for the realization phase. He is the initiator and Principal Investigator for this mission. Under his leadership, the DLR airborne SAR system, i.e., E-SAR, has been upgraded to operate in innovative imaging modes like polarimetric SAR interferometry and SAR tomography. He has more than 250 publications in international conferences and journals. He is the holder of 15 patents in the radar and antenna field. His professional interests and research areas encompass radar end-to-end system design and analysis, innovative microwave techniques and system concepts, signal processing, and remote sensing applications.

Prof. Moreira is a member of the IEEE Geoscience and Remote Sensing Society (GRS-S) Administrative Committee (1999-2001, 2004-2009, and 2010 as President), was the Founder and Chair of the German Chapter of the GRS-S (2003-2008), was an Associate Editor for the IEEE TRANSACTIONS ON Geoscience And Remote Sensing Letters (2003-2007), and has been serving as an Associate and Guest Editor for the IEEE TRANSACTIONS ON Geoscience And Remote Sensing since 2005. From 2003 to 2008, he served as a member of the Board of Directors of the Information Technology Society of the German Association for Electrical, Electronic and Information Technologies. He is currently the Chair of the Scientific and Technical Council of the DLR (2009-2011). He has contributed to the successful series of the European SAR conferences (EUSAR) since 1996 as a member of the Technical Program Committee, the Technical Chairman (2000), the Awards Chairman (2002-2004), the General Chairman (2006), and the Cochairman (2008). In 1995, he received the DLR Science Award. He and his colleagues received the GRS-S Transactions Prize Paper Awards in 1997, 2001, and 2007. He also received the IEEE Nathanson Award for the Young Radar Engineer of the Year in 1999 and the IEEE Kiyo Tomiyasu Field Award in 2007. 